\title{
NFKB is a critical transcriptional regulator of atypical cadherin FAT1 in glioma
}

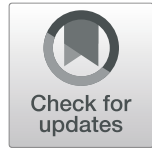

\author{
Chitrangda Srivastava ${ }^{1,2}$, Khushboo Irshad', Yakhlesh Gupta', Chitra Sarkar ${ }^{3}$, Ashish Suri ${ }^{4}$, \\ Parthaprasad Chattopadhyay', Subrata Sinha ${ }^{1}$ and Kunzang Chosdol ${ }^{1 *}$
}

\begin{abstract}
Background: Overexpression of FAT1 gene and its oncogenic effects have been reported in several cancers. Previously, we have documented upregulation of FAT1 gene in glioblastoma (GBM) tumors which was found to increase the expression of proinflammatory markers, HIF-1a, stemness genes and EMT markers in glioma cells. Here, we reveal NFKB (ReIA)/RelA/p65 as the transcriptional regulator of FAT1 gene in GBM cells.

Methods: In-silico analysis of FAT1 gene promoter was performed using online bioinformatics tool Promo alggen (Transfac 8.3) to identify putative transcription factor(s) binding motifs. A $4.0 \mathrm{~kb}$ FAT1 promoter $(-3220 \mathrm{bp}$ to +848 bp w.r.t. TSS + 1) was cloned into promoter less pGL3Basic reporter vector. Characterization of FAT1 promoter for transcriptional regulation was performed by in-vitro functional assays using promoter deletion constructs, site directed mutagenesis and ChIP in GBM cells.

Results: Expression levels of NFKB (RelA) and FAT1 were found to be increased and positively correlated in GBM tumors $(n=16)$, REMBRANDT GBM-database $(n=214)$ and TCGA GBM-database $(n=153)$. In addition to glioma, positive correlation between NFKB (ReIA) and FAT1 expression was also observed in other tumors like pancreatic, hepatocellular, lung and stomach cancers (data extracted from TCGA tumor data). A 4.0 kb FAT1-promoter-construct [-3220 bp/+ $848 \mathrm{bp}$, transcription start site (TSS) + 1, having $17 \mathrm{NFkB}$ (RelA) motifs] showed high FAT1 promoter luciferase-activity in GBM cells (U87MG/A172/U373MG). FAT1 promoter deletion-construct pGL3F1 [- 200 bp/+ 848 bp, with 3-NFkB (RelA)-motifs] showed the highest promoter activity. Exposure of GBM cells to known NFkB (RelA)activators [severe-hypoxia/TNF-a/ectopic-NFKB (RelA) + IKBK vectors] led to increased pGL3F1-promoter activity and increased endogenous-FAT1 expression. Conversely, siRNA-mediated NFkB (RelA) knockdown led to decreased pGL3F1-promoter activity and decreased endogenous-FAT1 expression. Deletion of NFkB (RelA)-motif at - $90 \mathrm{bp} /-$ 80 bp [pGL3F181-construct] showed significant decrease in promoter activity. Site directed mutagenesis at -90 bp/80 bp and ChIP assay for endogenous-NFkB (RelA) confirmed the importance of this motif in FAT1 expression regulation. Significant reduction in the migration, invasion as well as colony forming capacity of the U87MG glioma cells was observed on siRNA-mediated knockdown of NFKB (RelA).
\end{abstract}

Conclusion: Since FAT1 and NFKB (ReIA) are independently known to promote pro-tumorigenic inflammation and upregulate the expression of HIF-1a/EMT/stemness in tumors, targeting the NFKB (ReIA)-FAT1 axis may attenuate an important tumor-promoting pathway in GBM. This may also be applicable to other tumors.

Keywords: FAT1, NFKB (RelA), Glioblastoma, Tumor, Promoter

\footnotetext{
* Correspondence: kunzangchosdol@yahoo.com

${ }^{1}$ Department of Biochemistry, All India Institute of Medical Sciences, -110029,

New Delhi, India

Full list of author information is available at the end of the article
}

(c) The Author(s). 2020 Open Access This article is distributed under the terms of the Creative Commons Attribution 4.0 International License (http://creativecommons.org/licenses/by/4.0/), which permits unrestricted use, distribution, and reproduction in any medium, provided you give appropriate credit to the original author(s) and the source, provide a link to the Creative Commons license, and indicate if changes were made. The Creative Commons Public Domain Dedication waiver (http://creativecommons.org/publicdomain/zero/1.0/) applies to the data made available in this article, unless otherwise stated. 


\section{Background}

Glioblastoma (GBM) is the most aggressive form of glioma, resistant to almost all available treatment modalities [1-4]. The molecular alterations in GBM are wellcharacterized and the involvement of many oncogenic pathways and molecules including NFкB-pathway are known [1, 5-9]. NFкB is an important signaling molecule, upregulating pro-inflammatory and hypoxic responses that contribute to tumor pathology and resistance to therapy [8-10]. NFkB activation is known to promote tumor progression through mechanisms such as cell proliferation, apoptosis, angiogenesis, tumor metastasis, proinflammatory response, metabolic reprogramming etc. $[10,11]$. High constitutive NFKB activity is the characteristic of GBM $[9,10,12,13]$ and NFKB has been evaluated as a potential target for therapeutic intervention in GBM [12, 14]. NFKB is a known transcription factor, upregulating the expression of many oncogenic and pro-inflammatory molecules having crucial roles in the aggressive progression of different tumors including GBM $[10,11,15,16]$.

We have earlier reported the oncogenic role of the FAT1 gene in GBM. This is via the maintenance of a pro-inflammatory microenvironment [17], regulation of HIF- $1 \alpha$ via Akt/mTOR pathway [18] as well as by modulation of EMT/stemness molecules in hypoxic GBM [19]. FAT1, a transmembrane protein, is a non-classical proto-cadherin that has a role in developmental processes [20-22] as well as an oncogenic role in many human cancers like glioma, pancreatic cancer, leukemia, colon cancer, hepatocellular carcinoma (HCC) etc. [1719, 23-26]. In many cancers including glioma, FAT1 is known to increase migration and invasion of tumor cells $[17,18,27]$. Upregulated expression of FAT1 gene in different tumors including GBM have been reported [17$19,23,25,26]$ but till date, its upstream transcriptional regulation remains undefined.

In this study, in order to identify novel transcription factor(s) regulating FAT1 expression, we performed insilico analysis of the FAT1 promoter $(4.0 \mathrm{~kb})$ using online bioinformatics tools i.e. Promo alggen (Transfac 8.3). These showed multiple DNA binding motifs for more than 500 transcription factors (TFs), including NFKB, c-myc, Sp1, Elk-1, c-jun, c-fos, E2F. The NFкB family of transcription factors showed multiple DNA binding motifs with high probabilistic values on the FAT1 promoter. NFKB is one of the most potential and highly active transcription factors in GBM and a known master regulator of inflammatory signaling. It is a critical regulator of HIF-1 $\alpha$, a master regulator of tumor hypoxia $[28,29]$. We also observed increased expression and a positive association between NFKB (RelA) and FAT1 expression in GBM tumors. By in-vitro experiments like FAT1 promoter characterization and functional assays using GBM cell lines, we have been able to demonstrate the role of NFKB (RelA) as a potent transcriptional regulator of FAT1 expression. Hence, our study suggests an additional mechanism by which NFKB (RelA) can contribute to pro-tumorigenic microenvironment in GBM via FAT1.

\section{Methods}

\section{Aim and study design}

This study was aimed at identifying the transcription factor(s) regulating expression of FAT1 gene in glioma. For that, first we did in-silico identification of potential transcription factors binding on FAT1 promoter. Next, we did correlation analysis of the transcription factor, identified by in-silico analysis, and FAT1 expression in human GBM tumor samples obtained during study as well as in Rembrandt and TCGAGBM data sets. This was followed by in-vitro promoter characterization of FAT1 gene in glioma cells and functional role of NFкB (RelA) by analyzing migration/invasion and colony forming assay after siRNA mediated knockdown of NFкB (RelA) in U87MG glioma cells.

\section{In-silico analysis of FAT1 promoter}

The FAT1 gene is located on chromosome 4q35.2. The transcription start site (TSS) of FAT1 gene was identified by aligning $5^{\prime}$ upstream FAT1 transcript (Acc. No. NM_005245) with the human genome sequence using NCBI-BLAST tool. The online bioinformatics tool, PROMO software; (http://alggen.lsi.upc.es/cgi-bin/promov3/promo/promoinit.cgi?dirDB=TF8.3) was used to identify motifs for FAT1 transcriptional regulatory molecule(s) on the FAT1 promoter.

\section{GBM-tumor samples}

Surgically resected GBM samples $(n=16)$ were collected from Department of Neurosurgery/Neuropathology, AIIMS, New Delhi after approval by Institute EthicsCommittee, AIIMS, New Delhi (Ref.No IESC/T-416/ 01.11.2013). The histopathological diagnosis of the tumors was done by Prof Chitra Sarkar, Department of Pathology, AIIMS, New Delhi. Normal human brain total-RNA (Clontech, CA, USA) was used as control. REMBRANDT-GBM database (Affymetrix HG U133 v2.0 Plus) was accessed at http://caintegratorinfo.nci.nih. gov/rembrandt and analyzed for the expression of NFKB (RelA) and FAT1 genes in the available 214 GBM cases. In addition, TCGA expression data was also obtained from the open database available at http://www.proteinatlas.org for NFKB (RelA) and FAT1 expression in GBM as well as other tumors like pancreatic, hepatocellular, stomach and lung cancers. Gene expression data of RNA seq analyses was obtained in the form of FPKM values (data not normalized with respect to normal tissues) 
which was merged for NFKB (RelA) and FAT1 values and then subjected to Spearman's correlation analysis using GraphPad Prism Version 5.00.

\section{Cell culture}

Glioma cell lines (U87MG/A172/U373MG) were procured from ATCC (USA) and maintained in DMEM (Dulbecco's Modified Eagle's Medium, Hyclone), supplemented with $10 \%$ (v/v) FCS (fetal calf serum, Gibco), 3.7 $\mathrm{g} / \mathrm{l}$ sodium bicarbonate and ciprofloxacin $10 \mu \mathrm{g} / \mathrm{ml}$ and $5 \% \mathrm{CO}_{2}$ at $37^{\circ} \mathrm{C}$. The cells were cultured in $25 \mathrm{~cm}^{2}$ canted neck, vented cap tissue culture flasks, 6-well, 24well and 96-well plates in airtight chambers. Normoxic $\left(20 \% \mathrm{O}_{2}\right.$ and $\left.5 \% \mathrm{CO}_{2}\right)$ conditions were maintained using vacuum pump and gas proportionator (Anoxomat), which adjust the oxygen concentration by evacuating air and replacing it with nitrogen.

\section{PCR amplification and cloning of FAT1-promoter}

Genomic DNA was isolated from healthy human PBMCs using Qiagen kit (Germany), quantified and used to PCR amplify $\sim 4.0 \mathrm{~kb}$ FAT1 promoter using specific primers (sense and antisense primers; Additional file 1: Table S1) flanked with MluI and NheI sites for cloning purpose. Hot-start PCR was performed using pfu-Taq polymerase in the presence of $5 \%$ DMSO, the amplified product of a $4.0 \mathrm{~kb}$ FAT1 promoter sequence $(-3220 \mathrm{bp}$ to +848 bp w.r.t. TSS +1 ) was subjected to agarose gel electrophoresis, the amplicon was excised from the gel, purified and cloned in promoter less pGL3Basic reporter vector (Promega, promoter less vector). The vector with $4.0 \mathrm{~kb}$ cloned promoter was named pGL3F4. The pGL3F4 was transfected in DH5 $\alpha$ and plated. One of the positive colonies was confirmed by double digestion with MluI-NheI as well as with KpnI restriction enzyme to confirm the $5^{\prime}$ to $3^{\prime}$ directionality of insert. The confirmation of the cloned $4.0 \mathrm{~kb}$ FAT1 promoter (pGL3F4) was done by sequencing and DNA-BLAST analysis. The $4.0 \mathrm{~kb}$ FAT1 promoter was also analyzed forGC content and TATA/CAAT Box using Eukaryotic promoter database (EDP; http://epd.vital-it.ch/index.php).

\section{Sequential deletion of $4.0 \mathrm{~kb}$ FAT1 promoter to generate promoter deletion constructs}

The FAT1 promoter deletion constructs were generated by sequentially deleting the NFKB (RelA) motifs from the $5^{\prime}$ and the $3^{\prime}$ ends of the full length $4.0 \mathrm{~kb}$ promoter construct pGL3F4 [-3220 bp to $+848 \mathrm{bp}$ w.r.t. TSS] having 17-NFкB (RelA) binding motifs. The details of the FAT1-promoter constructs, number of NFкB (RelA) motifs present in each constructs and the sequences of the primers used are given in Additional file 1: Table S1 and Table S2.
By sequential deletion from $5^{\prime}$ end of the $4.0 \mathrm{~kb}$ promoter we generated (i) a promoter of $3.1 \mathrm{~kb}$ size [ $-2260 \mathrm{bp}$ to + 848 bp TSS] having 13-NFkB (RelA) binding motifs; (ii) a promoter of $2.0 \mathrm{~kb}$ size [ $-1230 \mathrm{bp}$ to +848 bp w.r.t. TSS] with 10-NFkB (RelA) binding motifs; and (iii) a promoter of $1.0 \mathrm{~kb}$ size [ $-200 \mathrm{bp}$ to +848 bp w.r.t. TSS] with 3-NFkB (RelA) binding motifs; by PCR amplification using specific primer pairs (Additional file 1: Table S1). The constructs were named as pGL3F3 (3.1 kb), pGL3F2 $(2.0 \mathrm{~kb})$ and pGL3F1 (1.0 kb), respectively. Further, from pGL3F2 (2.0 $\mathrm{kb}$ promoter) two more deletion constructs were generated viz. pGL3F2 $\delta 1$ of $1.3 \mathrm{~kb}$ [ $-530 \mathrm{bp}$ to +848 bp w.r.t. TSS] having 6-NFKB (RelA) motifs; and pGL3F2 22 of $1.1 \mathrm{~kb}$ [280 bp to +848 bp w.r.t. TSS] having 3-NFKB (RelA) motifs. And, from pGL3F1 $(1.0 \mathrm{~kb})$ two more deletion constructs were generated by $5^{\prime}$ and $3^{\prime}$ deletion to generate (i) pGL3F1 $\delta 1$ of $0.86 \mathrm{~kb}(-20 \mathrm{bp}$ to +848 bp w.r.t. TSS) with two NFKB (RelA) motifs at $+347 \mathrm{bp} /+356 \mathrm{bp}$ and $+360 \mathrm{bp} /$ $+368 \mathrm{bp}$ and (ii) pGL3F1 $\delta 2$ of $0.25 \mathrm{~kb}$ ( $-200 \mathrm{bp}$ to $+50 \mathrm{bp}$ w.r.t. TSS) with one NFкB (RelA) motif at $-90 \mathrm{bp} /-80 \mathrm{bp}$. The PCR-amplified products of different promoter lengths were subjected to agarose gel electrophoresis, the amplicons were gel extracted, purified using Promega PCRcleaning Kit (Promega, USA) following manufacturer's protocol and cloned in Luciferase expression-vector pGL3Basic (Promega, promoter less vector) at MluI/NheI sites. The sequences of all the deletion constructs were confirmed by sequencing and blast analysis.

\section{Promoter activity analysis by dual-luciferase assay}

U87MG, A172 and U373MG cells were cultured and plated in six-well plates. After overnight growth, the cells were transfected with the FAT1 promoter constructs using lipofectamine $2000^{\text {max }}$ reagent (Invitrogen, USA). After $48 \mathrm{~h}$ of transfection, Luciferase activity was analysed by Dual-luciferase assay kit (Promega, USA) following manufacturer's protocol.

\section{Overexpression and induction of NFKB (RelA) Severe-hypoxia treatment}

Glioma cells were exposed to severe hypoxia $\left(0.2 \% \mathrm{O}_{2}\right)$ and normoxia $\left(20 \% \mathrm{O}_{2}\right)$ using Anoxomat (Mart Chamber, Netherlands). Cells were transfected with pGL3F1 and exposed to severe hypoxia $\left(0.2 \% \mathrm{O}_{2}\right)$ for $48 \mathrm{~h}$, and lysates were prepared for promoter-activity analysis.

\section{Recombinant-human TNFa treatment}

Glioma cells were treated with recombinant human TNF $\alpha(100 \mathrm{ng} / \mathrm{ml})$ (Biolegend; Cat no. 570108) and harvested after $24 \mathrm{~h}$ for mRNA expression and promoteractivity analysis in pGL3F1-transfected cells. 


\section{NFKB (RelA)-overexpression vectors}

Glioma cells were transfected with NFкB (RelA)-expression vectors (RelA and IKBK/IKK vectors; eBABEpuro, kindly gifted by Prof. Soumen Basak, NII, New Delhi). The cells were harvested after $48 \mathrm{~h}$ of transfection for mRNA expression and promoter-activity analysis in pGL3F1 co-transfected cells.

\section{Knockdown of NFkB (RelA) expression}

Glioma cells were transiently transfected with $100 \mu \mathrm{M}$ of NFкB (RelA)-specific siRNA (ON-TARGETplus Human RelA siRNA-SMARTpool, Dharmacon) and $100 \mu \mathrm{M}$ control-siRNA (ON-TARGETplus Non-targeting Pool) for $48 \mathrm{~h}$ followed by mRNA expression and promoteractivity analysis in pGL3F1 co-transfected cells. Cell migration/invasion assay and anchorage-independent growth assays were performed after knockdown of NFKB (RelA) following the methods previously published [19].

\section{Expression analysis by qPCR}

Total RNA from treated and respective control cells was isolated using TRI reagent (Sigma, St Louis, MO). RNA quantification was done using spectrophotometer by measuring absorbance at 260 and $280 \mathrm{~nm}$. Total RNA $(1 \mu \mathrm{g})$ was used for reverse transcription using Revert Aid M-Mul-V Reverse transcriptase (MBI Fermentas, USA) and random decamers. For mRNA expression analysis, real time PCR was performed on Corbett Rotor Gene Q 6000 PCR machine. The details of primers used are listed in Additional file 1: Table S3.

\section{Western blot analysis}

U87MG cells were transfected with $1 \mu \mathrm{g}$ pBABE-RelA vector/control vector or with $100 \mu \mathrm{M} \mathrm{NF \kappa B} \mathrm{(RelA)-specific}$ siRNA/control-siRNA using lipofectamine $2000^{\text {max }}$ reagent (Invitrogen, USA) following manufacturer's protocol. After $48 \mathrm{~h}$ of transfection, whole cell lysates were prepared using RIPA buffer (Thermo Fisher Scientific) and protease inhibitor cocktail (Sigma-Aldrich). $40 \mu \mathrm{g}$ of the lysates were loaded in gradient SDS-PAGE gel (6-12\%), subjected to gel electrophoresis in Western blot apparatus (MiniPROTEAN Biorad gel apparatus) and transferred on to PVDF membrane. Primary antibodies used were: FAT1 (rabbit polyclonal, 1:500, Novus Biologicals) and $\beta$-actin (mouse monoclonal, 1:5000, Abkine). Densitometric analysis was done for the quantification of protein bands on the blot using ImageJ.

\section{Site directed mutagenesis (SDM)}

Site directed mutagenesis was done for the promoter reporter to elucidate the regulatory motif in FAT1 promoter by QuikChange II Site-Directed Mutagenesis Kit, according to the manufacturer's protocol. Primer details are given in Additional file 1: Table S3. Annealing temperature of the
SDM primers was standardized. pGL3F1 with the wild type NFKB (RelA) site was taken as the parental plasmid to amplify mutated construct using SDM primers (pGL3F1mtRelA) and confirmed by digestion of plasmid with DpnI restriction enzyme followed by gel electrophoresis. The plasmid was transformed and further confirmed by EcoRI digestion. The wild type vector (pGL3F1) and the mutated vector (pGL3F1mtRelA) were subjected to EcoRI digestion followed by gel electrophoresis. Confirmation of the mutated NFKB (RelA) sequence was done by sequencing of the wild type and the mutated vectors.

\section{Chromatin-immunoprecipitation (ChIP)}

U87MG cells were plated for $24 \mathrm{~h}$ and harvested for ChIP assay. Cells were fixed with $4 \%$ paraformaldehyde followed by sonication ( 1.5 cycle, $70 \%$ amplitude) to shear the gDNA. Sonicated lysate was pulled down with specific antibodies [Anti RelA Antibody, Anti pol-II (Positive control) and IgG (Negative control)]. Reverse cross-linking of DNA and protein was done for the PCR amplification using specific primers. Gel electrophoresis analysis confirmed the presence of ChIP product (FAT1) at $190 \mathrm{bp}$. ChIP assays were performed using ImprintTM Chromatin Immunoprecipitation Kit (Sigma-Aldrich, St. Louis, USA) according to the manufacturer's protocol. ChIP specific primers (ChIPF and ChIPR) were used to amplify FAT1 promoter region containing NFkB (RelA) transcription binding site (Additional file 1: Table S3).

\section{Transwell migration and invasion assay}

U87MG cells were harvested $48 \mathrm{~h}$ post-siRelA/siControl transfection, resuspended in serum-free medium and plated on top of transwell inserts $(8 \mu \mathrm{m}$ pore size $)$ in triplicate wells in 24-well plates. For invasion assay, $3 \times 10^{4}$ cells were seeded on inserts coated with matrigel, while for migration assay, $1 \times 10^{4}$ cells were seeded on uncoated inserts. Cells were allowed to migrate (for $24 \mathrm{~h}$ ) and to invade (for $48 \mathrm{~h}$ ) across transwell towards serum-containing medium at $37^{\circ} \mathrm{C}$. Cells inside the upper chamber were removed using cotton swabs. Migrated/invaded cells on the lower membrane surface were fixed in $4 \%$ paraformaldehyde, stained with DAPI, and counted microscopically at 20x magnification in five non-overlapping fields per well.

\section{Anchorage-independent colony formation assay}

In order to check the clonogenic ability of U87MG, cells were harvested $48 \mathrm{~h}$ post-siRelA/siControl transfection. A total of 15,000 cells/well were suspended in 0.3\% LMP agarose (Invitrogen) in DMEM supplemented with 20\% FBS and seeded on top of $0.6 \%$ agarose in 6-well plates in triplicates. $500 \mu \mathrm{l}$ of complete medium was added on top of the agarose layer twice a week. After 22 days in culture, the colonies were stained using $0.2 \%$ crystal violet. Mean number of colonies with $\geq 30 \mu \mathrm{m}$ diameter was 
counted microscopically at $4 \mathrm{x}$ magnification in ten nonoverlapping fields per well.

\section{Statistical analysis}

Parametric data results were expressed as means \pm S.E. (standard error) and comparisons were done by Student's $t$-test. For non-parametric data, results were expressed as mean \pm SEM (standard error of mean). A $p$ value $\leq 0.05$ was considered as statistically significant. Analysis of the GBM tumor data was done by Spearman's Rank Correlation Coefficient using GraphPad Prism Version 5.00.

\section{Results}

In-silico characterization of FAT1 promoter identifies multiple NFKB (RelA) binding motifs

To identify the binding motifs on the FAT1 promoter, first of all, the transcription start site (TSS) of FAT1 gene was identified by aligning $193 \mathrm{bp}$ of $5^{\prime}$ upstream FAT1 transcript (Acc. No. NM_005245) with the human genome using NCBI BLAST tool, which showed 100\% complementarily to the FAT1 gene (Accession numberNC_000004.12, www.ensembl.org) on chromosome 4q35.2 (Additional file 1: Fig. S1). A $4.0 \mathrm{~kb}$ FAT1 promoter sequence $[-3220 \mathrm{bp}$ to $+848 \mathrm{bp}$, with respect to transcription start site (w.r.t. TSS +1 )] was selected from Ensembl (Accession number-NC_000004.12) for the identification of binding motifs for FAT1 transcriptional regulatory molecule(s). Using the online bioinformatics tool, PROMO software; binding motifs for more than 500 transcription factors (TFs) were recognized with high (15\%) matrix dissimilarity. Binding motifs for multiple oncogenic TFs like NFкB, c-myc, sp1, Elk-1, c-jun etc. were noted on $4.0 \mathrm{~kb}-\mathrm{FAT} 1$ promoter. The full 4.0 kb FAT1 promoter-sequence with representative oncogenic TFs binding sites is depicted in Additional file 1: Fig. S2. Amongst all TFs, the NFKB family of transcription factors showed multiple binding sites (17 motifs) on FAT1 promoter with high matrix score (Additional file 1: Figs. S3a \& S3b). NFKB (RelA) is one of the most potent and active transcription factor of the NFKB family known to be associated with GBM [30]. Upregulated expression of FAT1 has been earlier found to be positively correlated with GBM aggressiveness [17-19, 31]. Our insilico finding of multiple $N F \kappa B$ (RelA) binding motifs on FAT1 promoter suggests $N F \kappa B$ (RelA) as a potential transcription factor in regulating FAT1 expression.

\section{A positive correlation between NFKB (RelA) and FAT1 expression in human GBM tumor samples}

To test the association of our in-silico finding of multiple NFKB (RelA) binding sites on FAT1 promoter, the correlation of expression of FAT1 and NFKB (RelA) was analyzed in GBM tumors $(n=16)$ obtained during the study. We observed increased expression of FAT1 in over $50 \%$ of GBM samples $(n=9 / 16)$ as compared to the expression in normal brain (Clontech, USA) (Fig. 1a). Spearman's correlation analysis displayed a strong positive correlation between the expression of NFKB (RelA) and FAT1 $(r=0.70 ; p=0.002)$ (Fig. 1b). REMBRANDT GBM database (Affymetrix HG U133 v2.0 Plus), obtained from publicly available Betastasis website, was analyzed for correlation between expression of $\mathrm{NFK}_{\mathrm{K}}$ (RelA) and FAT1. A significant positive Spearman's correlation was observed between the expression of $\mathrm{NFKB}$ (RelA) and FAT1 $(r=0.276 ; p=0.00004)$ (Fig. 1c). We observed significantly increased expression of $\mathrm{NFKB}$ (RelA) $(p<0.001)$ and FAT1 $(p<0.001)$ in GBM tumors $(n=214)$ as compared to normal brain $(n=20)$ (data not shown). A significant upregulation of FAT1 $(n=107$; $p=0.03)$ was observed in high NFKB (RelA) expressing GBM group as compared to low NFKB (RelA) expressing GBM group $(n=107)$, when GBM tumors were arranged in decreasing order of NFKB (RelA) expression (data not shown). We also checked the correlation of NFkB (RelA) and FAT1 expression in TCGA GBM data from the open database available on http://www.proteinatlas.org. A significant positive correlation $(r=0.213, p=0.008)$ was observed between NFkB (RelA) and FAT1 expression in GBM cases $(n=153)$ (Fig. 1d). Increased FAT1 mRNA expression in GBMs and its positive correlation with $\mathrm{NFKB}$ (RelA) expression pointed to a potential role of $\mathrm{NFK}_{\mathrm{K}}$ (RelA) as a transcriptional regulator of FAT1. In addition, a significant positive correlation between the NFkB (RelA) and FAT1 expression was observed in non-glioma tumors like pancreatic cancer $(n=176)$ cases $(r=0.307, p=$ $0.00003)$; hepatocellular cancer $(n=365)$ cases $(r=0.238$, $p=0.000004)$; stomach cancer $(n=354)$ cases $(r=0.158$, $p=0.003)$ and lung cancer $(n=994)$ cases $(r=0.126, p<$ $0.0001)$ (Additional file 1: Figure S4). The oncogenic role of FAT1 has been reported in these above cancers $[25,26$, $32]$ in addition to GBM [17-19]. Hence, the positive correlation between NFkB (RelA) and FAT1 expression extends to other tumors and is not exclusive to GBM.

\section{Characterization of the FAT1 promoter identifies regulatory NFKB (RelA) motif(s) in GBM cells}

The identification of multiple NFKB (RelA) binding motifs on FAT1 promoter and a positive correlation observed between FAT1 and NFKB (RelA) expression in GBM tumors provided a strong rationale for studying the transcriptional regulation of FAT1 by NFKB (RelA). To characterize the regulatory role of $\mathrm{NFK}_{\mathrm{K}}$ (RelA) as potential transcription factor, a $4.0 \mathrm{~kb} 5^{\prime}$ upstream sequence of FAT1 gene $(-3220 \mathrm{bp}$ to +848 bp w.r.t. TSS + 1) having seventeen (17) NFKB (RelA) binding motifs (Additional file 1: Figures S3a \& S3b) was cloned in pGL3Basic reporter vector (pGL3F4) (Fig. 2). The cloned 

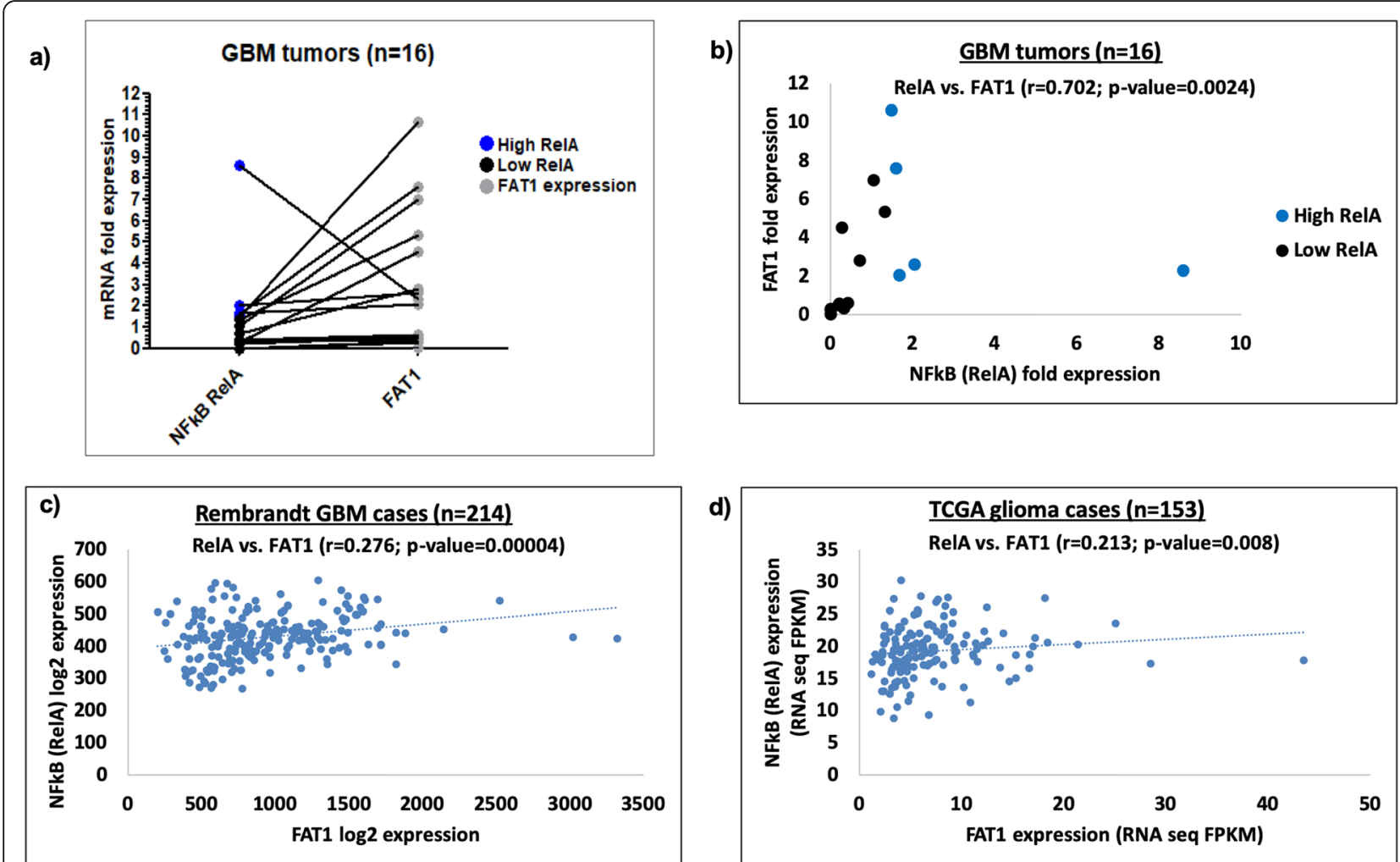

Fig. 1 a Expression analysis of NFkB (RelA) and FAT1 in GBM samples. mRNA expression of NFkB (RelA) and FAT1 genes was analyzed in GBM tumors ( $n=$ 16) with respect to the levels found in human normal brain total RNA (Clontech, USA) using qPCR. Increased expression of FAT1 was found in 9 out of 16 GBM samples as compared to the normal brain. High-NFkB expressors are represented as blue dots and low-NFkB expressors as black dots. FAT1 has been depicted with grey dots. $\mathbf{b}$ Correlation analysis of NFKB (RelA) and FAT1 expression in GBM samples. mRNA fold expression values of NFKB (RelA) and FAT1 found in the sixteen GBM tumors were correlated using Spearman's analysis by SPSS 11.5. A significant positive correlation was observed between NFKB (RelA) and FAT1 ( $r=0.7, p=0.002)$ in the studied samples. GBM samples have been displayed as high NFkB (RelA) expressors (blue dots) and low NFkB (RelA) expressors (black dots) on the basis of a cut-off value of $\geq 1.5$ fold expression. c Correlation of NFkB (RelA) and FAT1 expression in Rembrandt GBM database. Expression values of NFKB (RelA) and FAT1 were obtained and correlated in 214 GBM cases belonging to Rembrandt database. A significant positive correlation was observed between NFKB (RelA) and FAT1 (Spearman's ' $r$ ' $=0.276, p=0.00004$ ) in the analyzed cases. $\mathbf{d}$ Correlation of NFKB (RelA) and FAT1 expression in TCGA glioma database. Expression values of NFKB (RelA) and FAT1 were obtained and correlated in 153 glioma cases belonging to TCGA database. A significant positive correlation was observed between NFKB (RelA) and FAT1 (Spearman's ' $r=0.213, p=0.008$ ) in the analyzed cases

$4.0 \mathrm{~kb}$ promoter sequence was confirmed by sequencing and BLAST analysis (Additional file 1: Figure S5). The FAT1 promoter has been found to have high GC content in the core promoter but lacks the TATA Box and CAAT Box in its promoter (Additional file 1: Figure S6).

As per earlier reports of high $\mathrm{NF}_{K} \mathrm{~B}$ (RelA) [10] and FAT1 expression [17] in GBM cells, high relative mRNA expression of FAT1 and NFKB (RelA) was observed in GBM cells with the highest level in U87MG followed by A172 and U373MG (Fig. 3a). The $4.0 \mathrm{~kb}$ promoter construct (pGL3F4) was transfected in GBM cells (U87MG, A172 and U373MG) and luciferase activity was analysed by dual luciferase assays after $48 \mathrm{~h}$ of transfection. Increased luciferase activity of pGL3F4, normalized to the promoter less vector-control pGL3Basic, was observed in all GBM cells, with the highest activity in U87MG (8.41 fold \pm 0.70 ) followed by A172 (4.73 fold \pm 0.14 ) and
U373MG (3.57 fold \pm 0.23 ), as compared to their respective controls (Fig. 3b).

To characterize the potential NFkB (RelA) motif(s) on the FAT1 promoter, important for FAT1 expression regulation, a total of seven deletion constructs were generated (Fig. 4a) by sequentially deleting the NFKB (RelA) motifs at $5^{\prime}$ and $3^{\prime}$ ends of FAT1 promoter (pGL3F4) construct. The deletion constructs pGL3F3 [with 13-NFkB (RelA) motifs], pGL3F2 [with 10-NFKB (RelA) motifs] and pGL3F1 [with 3-NFKB (RelA) motifs] were transfected in U87MG cells for $48 \mathrm{~h}$. Luciferase activity was analysed along with the parent construct pGL3F4 [having 17-NFKB (RelA) motifs]. Among all promoter constructs, pGL3F1 showed the highest activity ( 18.29 fold \pm 2.76 ), followed by pGL3F4 (7.14 fold \pm 0.61 ), pGL3F3 (3.06 fold \pm 0.21 ) and pGL3F2 (0.29 fold \pm 0.02$)$ as compared to pGL3basic empty vector (Fig. 4b). The reduced promoter activity of 


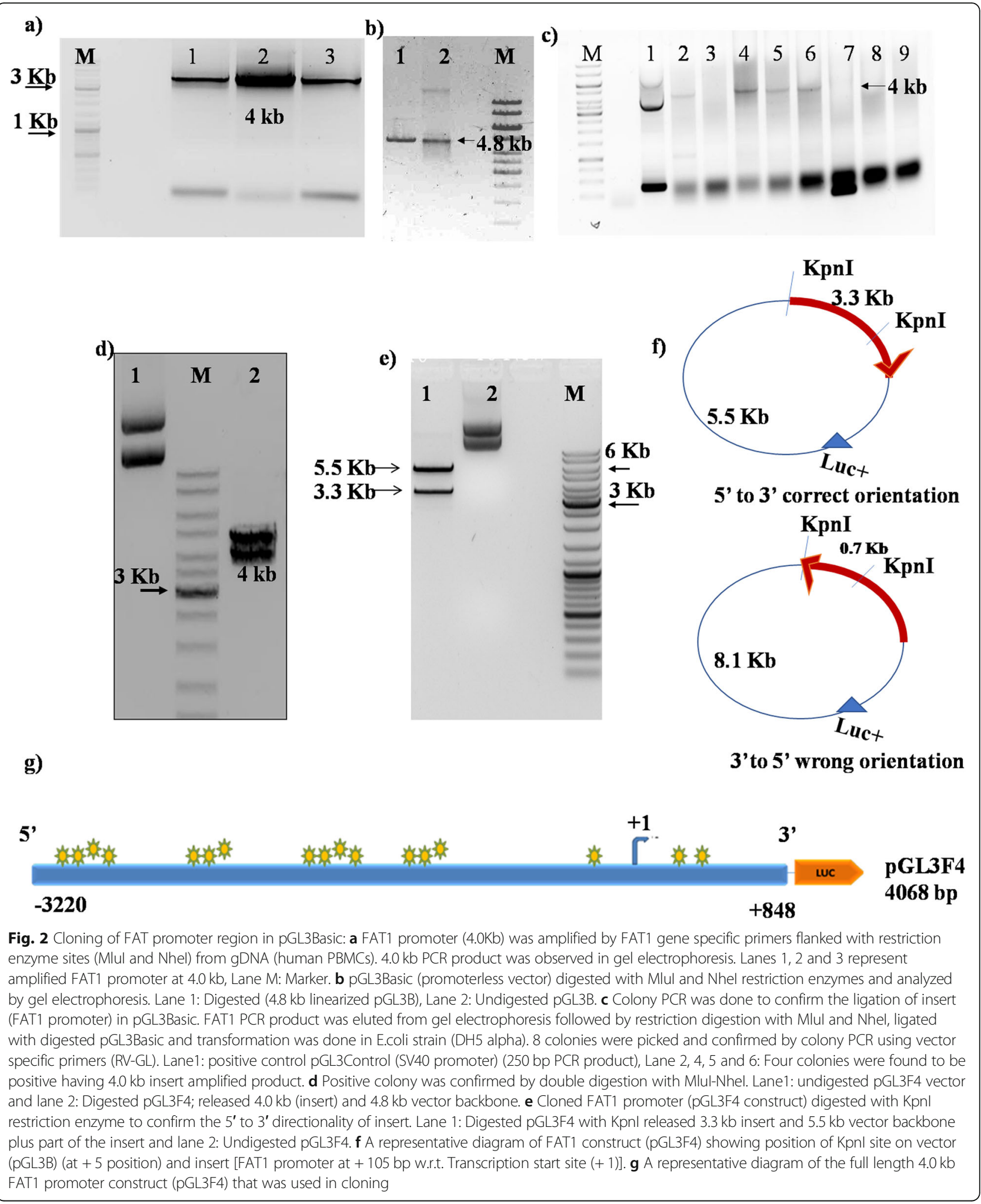

pGL3F2 could be due to the binding of trans-repressors as identified in our in-silico analysis. However, in this study we have focused on regulation of FAT1 expression by trans-activator [e.g. NFкB (RelA)].
To narrow down the regulatory NFKB (RelA) motif(s), we further deleted NFKB (RelA) motif(s) from $5^{\prime}$ and 3'ends of FAT1 promoter construct pGL3F1 with three NFкB motifs to generate (i) pGL3F181 [with two NFкB 
a)

\section{FAT1 and NFkB(RelA) expression in glioma cell lines}

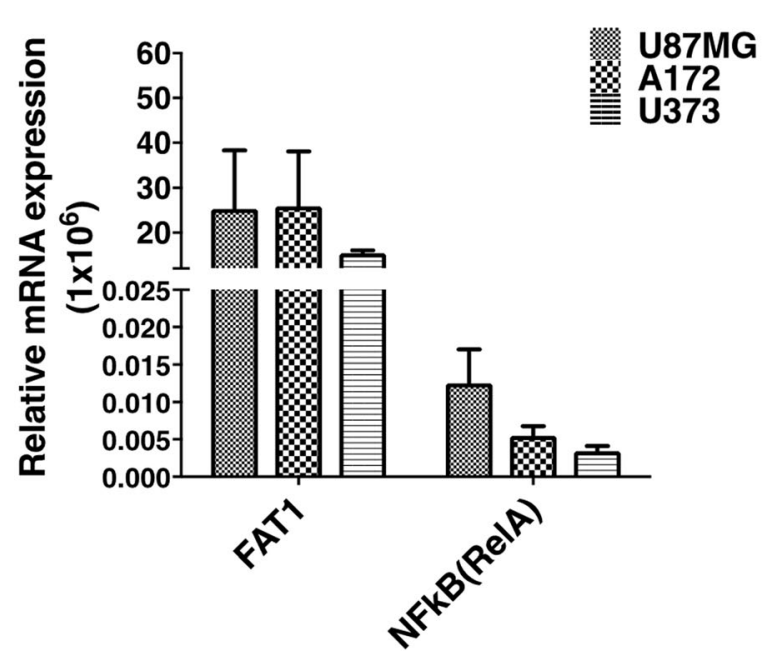

b)

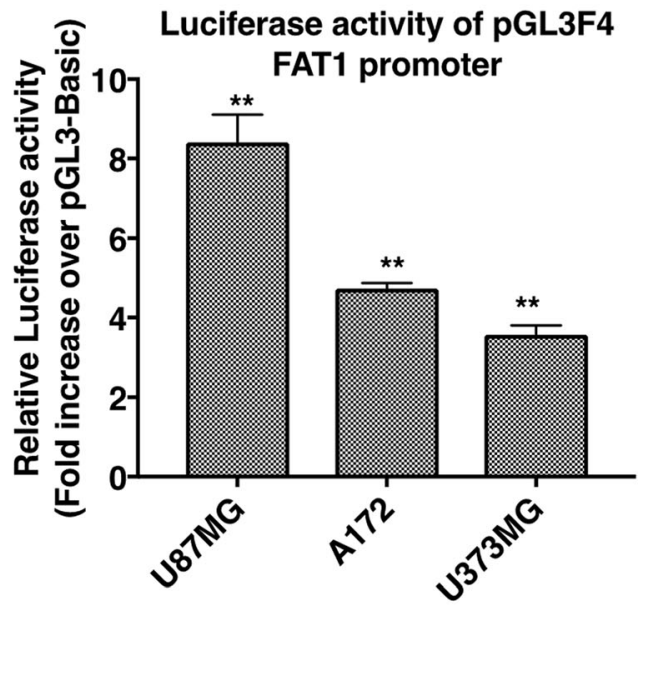

Fig. 3 a Expression of FAT1 and NFKB (ReIA) in glioma cell lines. Increased relative mRNA expression of FAT1 \& NFKB (ReIA) in U87MG, A172 and U373MG was observed. b Luciferase activity of full length 4.0 kb FAT1 promoter (pGL3F4) in glioma cell lines. Glioma cell lines (U87MG, A172 and U373MG) were transfected with pGL3F4 and pGL3B (promoterless vector). Luciferase activity was analysed by dual luciferase assay after $48 \mathrm{~h}$ of transfection. Significant increase in luciferase activity of PGL3F4 was found, with U87MG showing the highest luciferase activity followed by A172 and U373MG

(RelA) motifs] and (ii) pGL3F182 with one NFKB (RelA) (Fig. 4a). The luciferase activity of these constructs (pGL3F1/pGL3F181/pGL3F182) was analyzed in U87MG cells. We observed comparable promoter activity of pGL3F1 and pGL3F1 22 with 18 fold \pm 0.61 and 19.65 fold \pm 1.78 , respectively. Interestingly, pGL3F1 $\delta 1$, with deleted NFKB (RelA) site at $-90 \mathrm{bp} /-80 \mathrm{bp}$, showed significant reduction in the promoter activity (4.43 fold \pm 0.61 ) as compared to pGL3F1 (Fig. 4c).

We also generated two more deletion constructs pGL3F2 $\delta 1$ with 6-NFkB (RelA) motifs and pGL3F2 22 with 3 -NFkB (RelA) motifs from pGL3F2 construct (Fig. 4a). The promoter constructs pGL3F2 $\delta 1$ and pGL3F2 $\delta 2$ have shown higher luciferase activity than the parent construct pGL3F2. However, the luciferase activity observed in these constructs pGL3F2/pGL3F2 $1 /$ pGL3F2 $\delta 2$ was lower than pGL3F1 (Additional file 1: Figure S7).

The above findings suggested that the potential FAT1 promoter region ( $p$ GL3F1) from $-200 b p$ to $+848 b p$ w.r.t. $T S S(+1)$ and NFKB (RelA) binding motif at $-90 \mathrm{bp} /-80 \mathrm{bp}$ might be a key regulatory site to control FAT1 expression in GBM cells.

NFkB (RelA) upregulates FAT1 promoter (pGL3F1 construct) activity and endogenous expression in GBM Since the potential FAT1 promoter region is from -200 bp to +848 bp w.r.t. TSS $(+1)$, we further validated the role of NFKB (RelA) on FAT1 promoter (pGL3F1) activity and expression of endogenous-FAT1 mRNA in U87MG cells by increasing the NFKB (RelA) levels in the cells by exposing to known NFkB (RelA) modulators.

\section{Severe hypoxia $\left(0.2 \% \mathrm{O}_{2}\right)$ upregulates FAT1 promoter} activity and endogenous FAT1 expression

Since hypoxia is known to positively upregulate $\mathrm{NFKB}$ pathway $[15,16,33]$, U87MG cells transfected with FAT1 promoter construct-pGL3F1 which had the highest promoter activity were exposed to severe hypoxia $\left(0.2 \% \mathrm{O}_{2}\right)$ for $48 \mathrm{~h}$. The promoter activity was measured and compared to pGL3F1 transfected cells exposed to normoxia $\left(20 \% \mathrm{O}_{2}\right)$. There was significant increase (9.5 fold \pm 0.37 ) in pGL3F1 activity on exposure to severe hypoxia as compared to normoxia (Fig. 5a). In addition, U87MG and A172 showed increased endogenous-FAT1 expression [ 4.97 fold \pm 0.82 and 4.38 -fold \pm 0.38 in hypoxic cells $(0.2 \%$ $\mathrm{O}_{2}, 48 \mathrm{~h}$ ) respectively] as compared to the respective normoxic cells (Fig. 5b). The increase in NFKB (RelA) transcript levels in U87MG and A172 was 1.91 -fold \pm 0.36 and 1.97 -fold \pm 0.36 respectively on severe hypoxia exposure (Fig. 5b). This suggests the positive influence of hypoxiaupregulated NFkB (RelA) on FAT1 promoter activity.

\section{TNFa upregulates FAT1 promoter activity and endogenous FAT1 expression}

TNF $\alpha$ causes phosphorylation of IKB $\alpha$ (Inhibitor of $\mathrm{NF} \mathrm{B}$ ) followed by proteosomal degradation, leading to 
a)

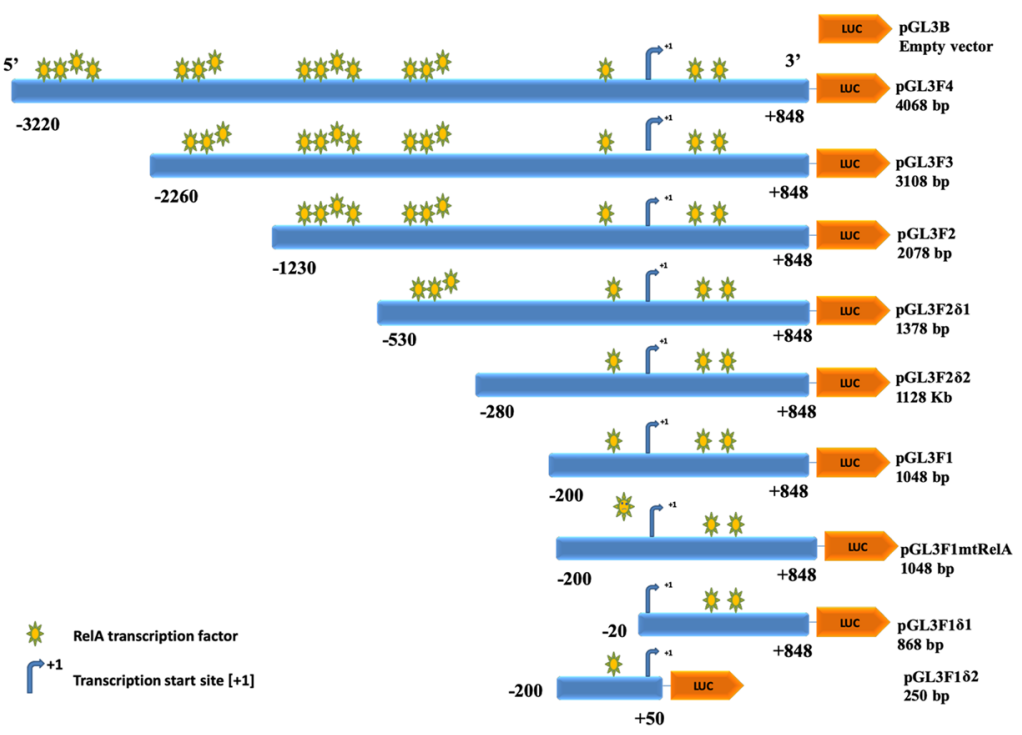

b)

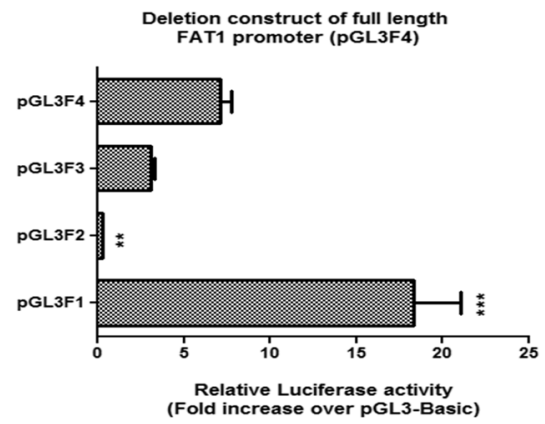

c)

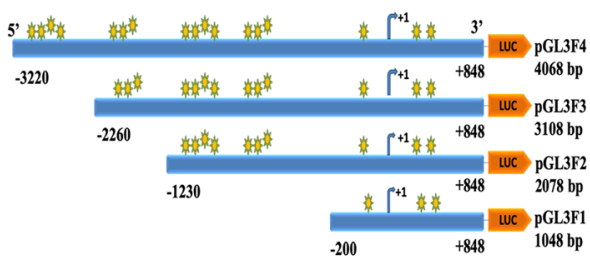
(Fold increase over pGL3-Basic)

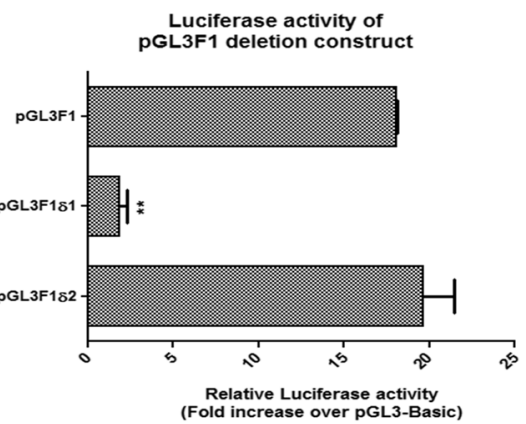

Fig. 4 (See legend on next page.) 
(See figure on previous page.)

Fig. 4 a A schematic diagram of FAT1 promoter constructs. 5' and 3' deletion constructs were generated from 4.0 kb full length FAT1 promoter (-3220/+ 848 bp). To amplify FAT1 promoter region ( $4.0 \mathrm{~kb}$ ) primers were designed (using Primer 3 software) and $5^{\prime}$ flanked with restriction enzymes, Mlul in forward primer and Nhel in reverse primer. To amplify 5 ' sequentially deleted FAT1 construct, forward primers were designed at the corresponding sites (at $-2260 \mathrm{bp},-1230 \mathrm{bp},-530 \mathrm{bp},-280 \mathrm{bp},-200 \mathrm{bp},-20 \mathrm{bp}$ ) of the respective FAT1 constructs [- $3220 \mathrm{bp} /+848 \mathrm{bp}$ w.r.t. TSS (+ 1)] while the reverse primer (R) (at + 848 bp) was constant. To amplify 3' deletion FAT1 construct, reverse primer was designed at corresponding site (at $+50 \mathrm{bp}$ ) of respective FAT1 construct and forward primer (at -200 bp) was paired. The FAT1 promoter inserts (F4, F3, F2, F2 $\delta 1, F 2 \delta 2, F 1, F 1 \delta 1$ and F1 $\delta 2$ ) display TSS (+ 1), size of insert, 5' upstream and $3^{\prime}$ downstream region of FAT1 promoter and yellow-green stars showing binding sites of NFkB (RelA) on each construct. b Luciferase activity of 5' deletion constructs (pGL3F3, pGL3F2 and pGL3F1) of FAT1 promoter (pGL3F4). U87MG cells were transfected with pGL3F4 (4.0 kb) and its 5'deletion constructs [pGL3F3 (3.1 kb), pGL3F2 (2.0 kb) and pGL3F1 $(1.0 \mathrm{~kb})]$ for $48 \mathrm{~h}$ followed by dual luciferase assay. pGL3F1 showed the highest luciferase activity followed by pGL3F4, pGL3F3 and pGL3F2 as compared to pGL3B. c Luciferase activity of 5' and 3' deletion constructs of FAT1 promoter construct (pGL3F1): FAT1 promoter constructs [pGL3F181 (0.86 kb) and pGL3F182 (0.25 kb)] and pGL3F1 were transfected in U87MG; and luciferase activity was analyzed after $48 \mathrm{~h}$. A comparable luciferase activity was observed in pGL3F1 and pGL3F1 $\delta 2$. A significant decrease in the luciferase activity was observed in pGL3F1 1 (4.43 fold \pm 0.61 ) as compared to pGL3F1. Bar graph represents fold luciferase activity of FAT1 promoter constructs (normalized to pGL3Basic and internal control $p R L T K)$. Values are mean \pm SE of at least three independent experiments performed in triplicates. Statistical analysis was performed using a paired two-tailed Student's $t$-test. $P$-value $<0.05$ was taken as significant $\left({ }^{*} p<0.05,{ }^{* *} p<0.01,{ }^{* * *} p<0.001\right)$. Generated constructs were cloned and confirmed by sequencing and BLAST analysis

release of the transcription factor $\mathrm{NFKB}$ (RelA) and its nuclear transport $[13,30]$. To check for the effect of TNF $\alpha$ treatment on FAT1 promoter activity, U87MG cells were transfected with FAT1 promoter (pGL3F1 construct) followed by TNF $\alpha(100 \mathrm{ng} / \mathrm{ml})$ treatment every $24 \mathrm{~h}$ for $48 \mathrm{~h}$ followed by measurement of pGL3F1 promoter activity. There was significant increase in pGL3F1 promoter activity (7.03-fold \pm 0.08$)$ in cells on TNF $\alpha$ treatment as compared to TNF $\alpha$ untreated cells (Fig. 5c). U87MG and A172 cells treated with TNF $\alpha$ $(100 \mathrm{ng} / \mathrm{ml})$ for $24 \mathrm{~h}$ also showed increased endogenous FAT1 mRNA expression by 2.02 -fold \pm 0.09 and 1.72 -

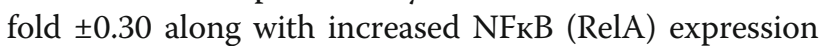
by 1.76 -fold \pm 0.21 and 2.24 -fold \pm 0.03 respectively as compared to TNF $\alpha$ untreated control cells (Fig. 5d).

\section{Exogenous expression of NFKB (RelA) upregulates FAT1 promoter activity and endogenous FAT1 expression}

Next, we overexpressed NFKB (RelA) by co-transfection of RelA vector [NFkB (RelA) transcription factor- cDNA cloned in eBABE vector] and it transcriptional activator, IKBK construct (IKK/NFKB kinase- cDNA cloned in eBABE vector) (constructs were kindly gifted by Dr. Soumen Basak, NII, New-Delhi) in glioma cells. This was followed by analysis of FAT1 promoter activity and endogenous FAT1 transcript level.

U87MG cells were co-transfected with pGL3F1 + RelA+IKBK constructs or pGL3F1 + control vectors (pGL3B + eBABE vectors) and analyzed for FAT1 promoter activity $48 \mathrm{~h}$ post-transfection. Cells transfected with NFKB (RelA) (RelA+IKBK vector) showed significantly increased FAT1 promoter (pGL3F1) activity (2.22fold \pm 0.21 ) as compared to control cells (transfected with empty-vectors) (Fig. 5e). We also observed increased mRNA expression of endogenous-FAT 1 by 2.52 -fold \pm .20 and 1.55 -fold \pm 0.06 in U87MG and A172 cells respectively along with increased NFKB (RelA) level by 1745.9 -fold \pm
21.9 and 695.0-fold \pm .68 .04 fold in U87MG and A172 respectively in NFKB (RelA) overexpressed cells as compared to control cells (empty vectors) (Fig. 5f).

\section{NFKB (RelA) abrogation downregulates FAT1 promoter activity and endogenous FAT1 expression in GBM cells} The role of NFKB (RelA) in regulating FAT1 expression has been further validated by abrogating NFкB (RelA) level by using NFKB (RelA) specific siRNA in GBM cells. U87MG and A172 cells were transfected with $100 \mu \mathrm{M}$ of NFKB (RelA) specific siRNA (siRelA) and control siRNA (siControl) for $48 \mathrm{~h}$ and observed 63 and 68\% knockdown of NFKB (RelA) in U87MG and A172 respectively (Fig. 6a). On NFKB (RelA) knockdown, there was significant reduction in the expression of FAT1 in U87MG (55\%-reduction) and A172 (52\%-reduction). In addition, the FAT1 promoter activity (pGL3F1) was significantly $(p \leq 0.05)$ reduced in siRelA treated cells as compared to siControl treated cells, normalized to pGL3Basic (Fig. 6b). Similarly, FAT1 reduction was observed at protein level on NFkB (RelA) knockdown in U87MG cells and increased FAT1 was observed at protein level on overexpression of NFKB (RelA) in U87MG cells (Fig. 6c). Hence, these results confirm the regulatory effect of $N F \kappa B$ (RelA) on FAT1 promoter activity as well as on the endogenous-FAT1 expression in GBM cells.

\section{Site directed mutagenesis and chromatin Immunoprecipitation (ChIP) assay confirm the functional association of NFKB (RelA) with FAT1 promoter}

The FAT1 promoter deletion analysis of pGL3F1 suggests that NFKB (RelA) motif at -90/- 80 bp (pGL3F1 $\delta 1$, with deleted NFkB (RelA) site at $-90 \mathrm{bp} /-80 \mathrm{bp}$ ) may be the most potential regulatory site on FAT1 promoter (Fig. 4c). Hence, to confirm its role in FAT1 expression a site directed mutagenesis was carried out at this site of NFKB (RelA) at $-90 \mathrm{bp} /-80 \mathrm{bp}$ in pGL3F1 promoter 


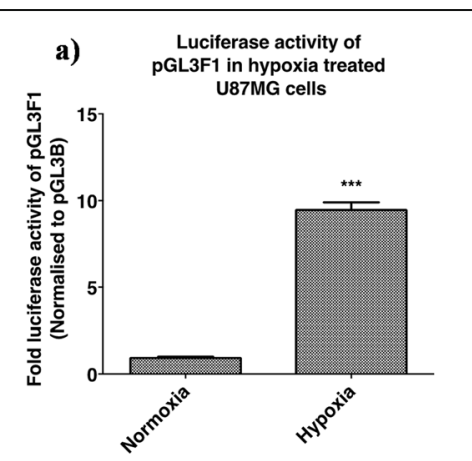

b)

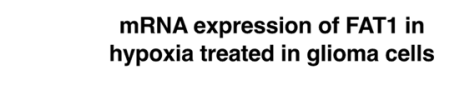

c)

Luciferase activity of pGL3F1 in TNF $\alpha$ treated

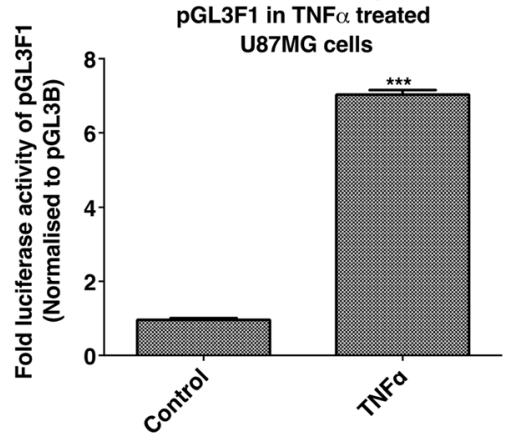

e)

Luciferase activity of pGL3F1 in NFkB(RelA)
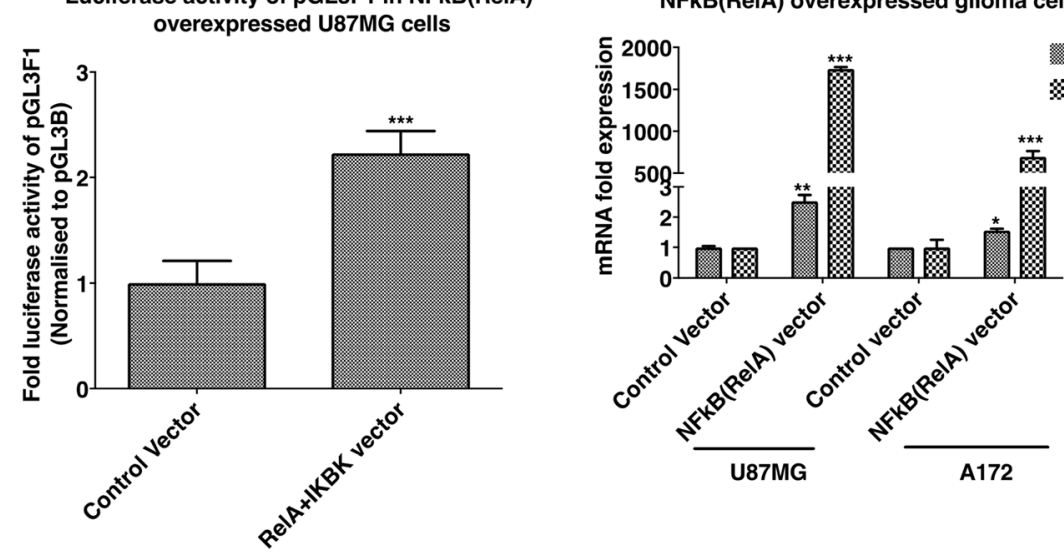

은 8

\& NFkB(RelA)
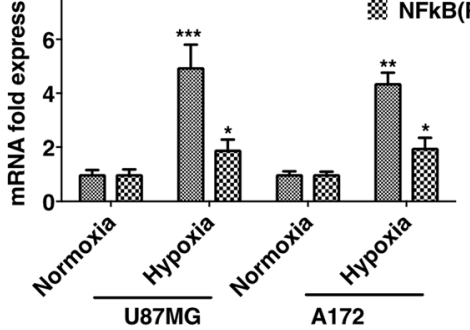

d)

mRNA expression of FAT1 in

TNF $\alpha$ treated in glioma cells

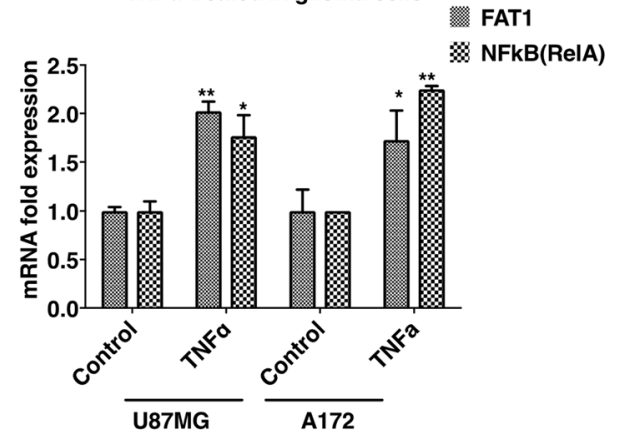

f)

mRNA expression of FAT1 in

NFkB(RelA) overexpressed glioma cells

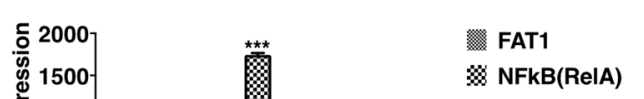

Fig. 5 Effect of NFKB pathway activation on FAT1 promoter activity and endogenous FAT1 expression. NFkB pathway activation was done by treating GBM cells with known NFKB activators (severe hypoxia, TNFa and NFKB expression vector). a FAT1 promoter activity under severe hypoxia. GBM cells transfected with pGL3F1 were exposed to severe hypoxia $\left(0.2 \% \mathrm{O}_{2}\right)$ and normoxia $\left(20 \% \mathrm{O}_{2}\right)$ for $48 \mathrm{~h}$. Luciferase activity of pGL3F1 was significantly increased under severe hypoxia as compared to normoxic control U87MG. $\mathbf{b}$ FAT1 mRNA expression under severe hypoxia. Glioma cells exposed to severe hypoxia showed significant increase in FAT1 mRNA expression along with increased NFkB (RelA) expression in both U87MG and A172 as compared to the respective normoxic cells. c FAT1 promoter activity in TNFa treated glioma cells. GBM cells were transfected with pGL3F1 and treated with TNFa $(100 \mathrm{ng} / \mathrm{ml})$ for $24 \mathrm{~h}$ followed by FAT1 promoter activity and mRNA expression analyses. Significant increase in PGL3F1 luciferase activity was observed in U87MG cells treated with TNFa as compared to the control (untreated cells). d FAT1 mRNA expression in TNFa treated glioma cells. Glioma cells were treated with TNFa as described above. Increased endogenous FAT1 mRNA expression in both U87MG and A172 was seen along with increased NFKB (RelA) expression, compared to the untreated control cells. e FAT1 promoter activity in glioma cells with overexpressed NFKB (ReIA). GBM cells were co-transfected with pGL3F1 + RelA+IKBK and control vector ( $\mathrm{pGL} 3 \mathrm{~B}+\mathrm{eBABE}$ ) for $48 \mathrm{~h}$ followed by FAT1 promoter activity and mRNA expression analyses. Significantly increased FAT1 promoter activity was observed in the cells co-transfected with RelA+ IKBK expression vector as compared to the control cells. $\mathbf{f}$ FAT1 mRNA expression in glioma cells with overexpressed NFKB (RelA). U87MG and A172 cells co-transfected with RelA+ IKBK showed increased mRNA expression of FAT1 and NFkB (RelA) expression as compared to the control cells. Bar graph represents fold luciferase activity of FAT1 promoter constructs (normalized to pGL3Basic and internal control pRLTK) and fold mRNA expression. All experiments were done in triplicates and repeated thrice. Statistical analysis was performed using a paired two-tailed Student's $t$-test. $P$-value $<0.05$ was taken as significant $\left(^{*} p<0.05\right.$,

$\left.{ }^{* *} p<0.01,{ }^{* *} p<0.001\right)$ 


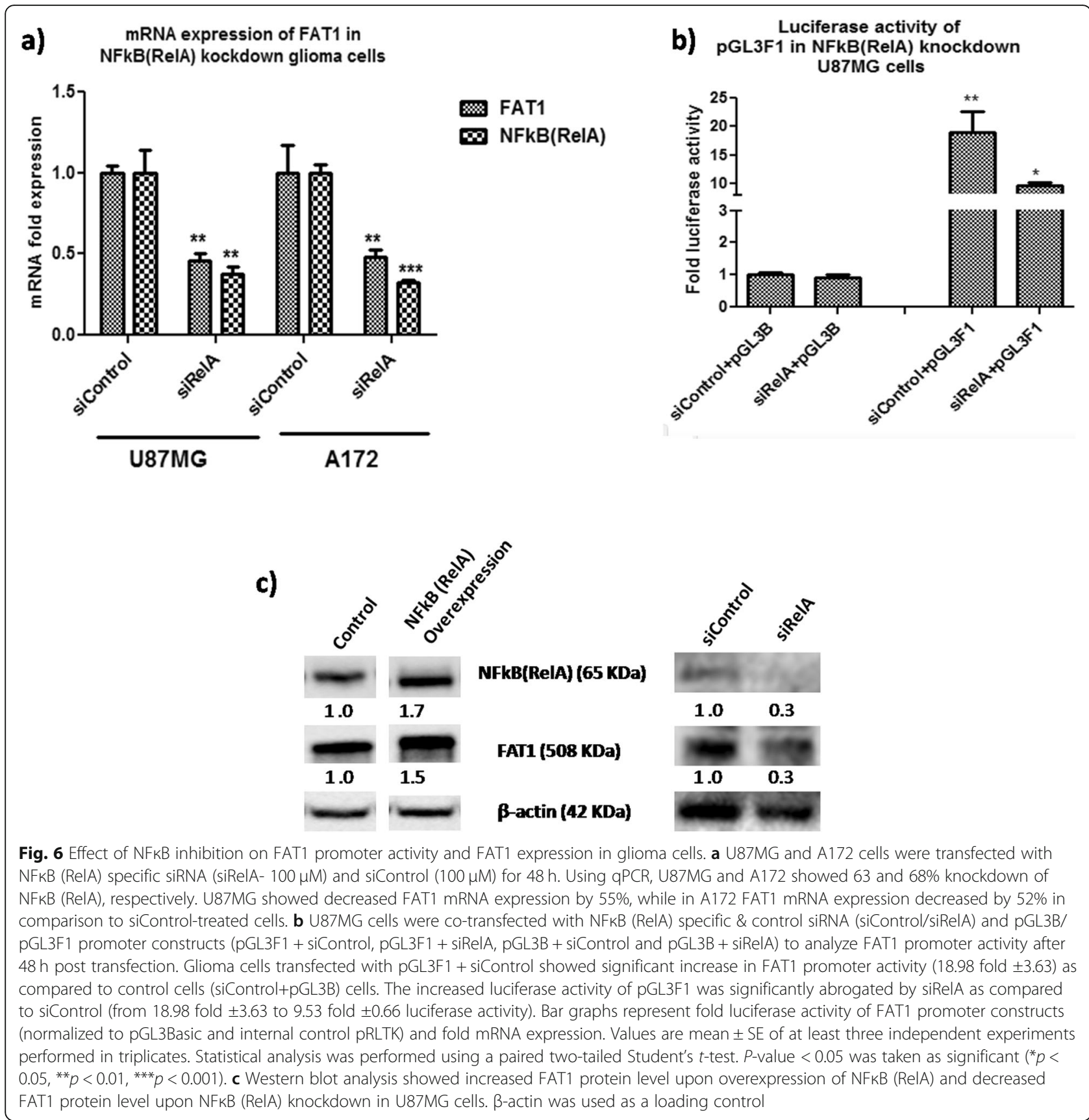

construct (Wild RelA site: 5'GGGGAAAGTG3': Mutated RelA site: 5'GAATTCAGTG3'). Blast analysis was done to confirm the sequence specificity of wild type (with Matrix score-11.16 for NFKB (RelA) and mutated (non binding) NFKB (RelA) binding site by Promo software (Fig. 7a). PCR amplification of mutated RelA construct (pGL3F1mtRelA) from wild type construct (pGL3F1) was done by using QuikChange II SiteDirected Mutagenesis Kit (Additional file 1: Figure S8) and confirmed by EcoRI digestion and sequencing (Additional file 1: Figure S9).
U87MG cells were transfected with pGL3F1 construct [wild type NFKB (RelA) site at $-90 \mathrm{bp} /-80 \mathrm{bp}$ ] and pGL3F1mtRelA construct [mutated NFKB (RelA) site at $-90 \mathrm{bp} /-80 \mathrm{bp}$ ] and $48 \mathrm{~h}$ post transfection the promoter activity was analyzed. We observed a significant decrease $(p<0.01)$ in the promoter activity in mutated pGL3F1mtRelA construct as compared to the wildtype pGL3F1 construct (Fig. 7b).

In order to confirm the specificity of $\mathrm{NFKB}$ (RelA) site, we also analysed the activity of both wild-type and mutated FAT1 promoters (pGL3F1 and pGL3F1mtRelA, 


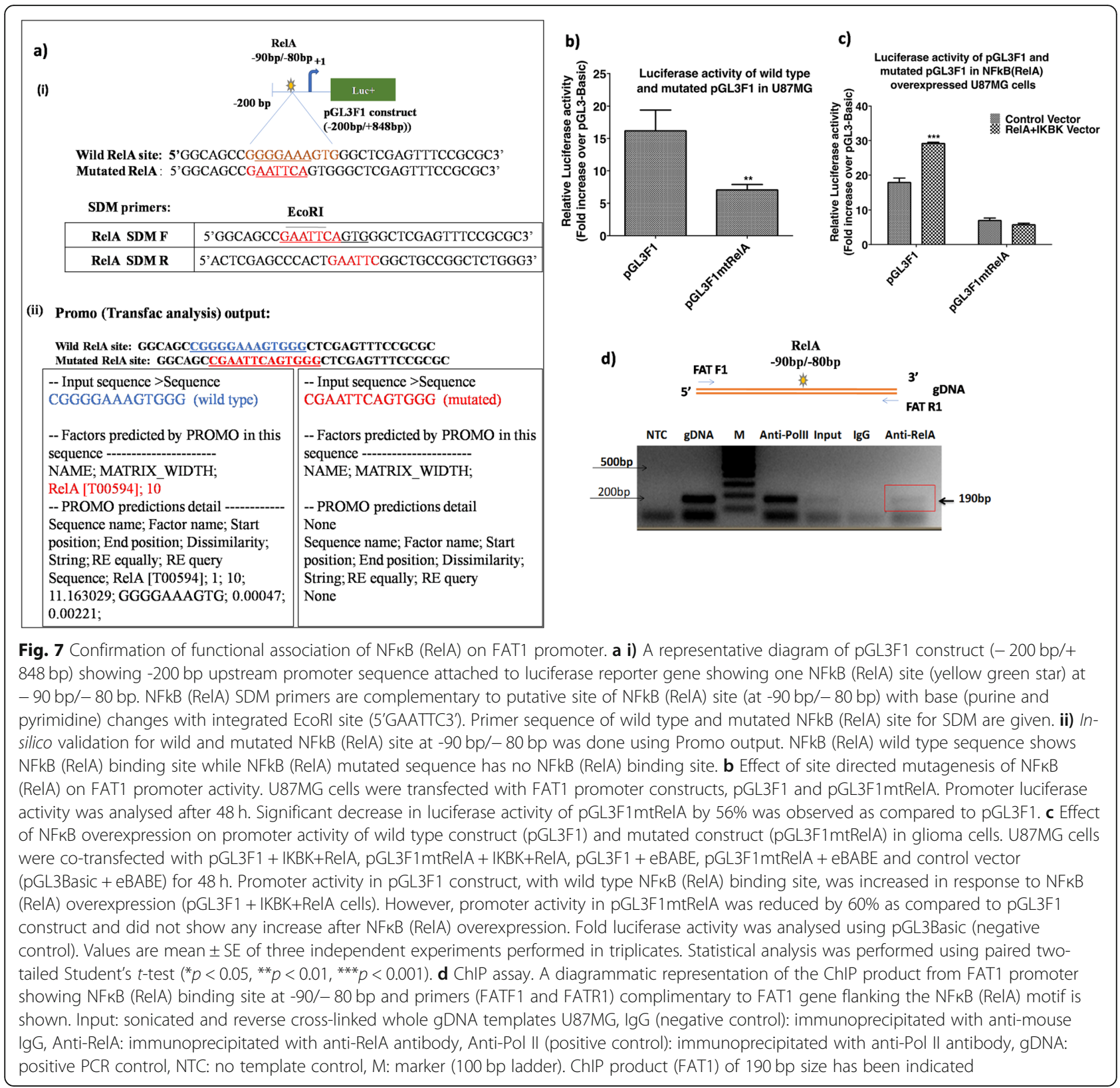

respectively) in cells overexpressed with of NFKB (RelA) by co-transfecting RelA+IKBK expression vectors and compared with vector-control (empty vector-eBABE) treated cells. The relative luciferase activity of wild type FAT1 promoter (pGL3F1) was significantly increased ( $p<$ 0.01 ) to 29.33 -fold \pm 0.15 in NFKB (RelA) overexpressing cells (co-transfected with RelA+IKBK vectors) as compared to 18.08 -fold \pm 1.05 in control cells (i.e. eBABE vector) (Fig. 7c).

Also, promoter luciferase activity in the construct pGL3F1mtRelA with mutated NFkB (RelA) binding site was significantly decreased by $60 \%$ as compared to the
pGL3F1 construct with wild type NFKB (RelA) binding site and it did not show any increase in the luciferase activity after NFKB (RelA) overexpression (IKBK+RelA vectors) (Fig. 7c).

After confirming the regulatory role of $\mathrm{NFK}_{\mathrm{K}}$ (RelA) motif $-90 \mathrm{bp} /-80 \mathrm{bp}$, by site directed mutagenesis, ChIP assay was performed to confirm the binding of endogenous NFkB (RelA) at $-90 \mathrm{bp} /-80 \mathrm{bp}$ site on FAT1 promoter. For that, U87MG cells were plated and cultured under standard culture conditions for $24 \mathrm{~h}$, cells were fixed with $4 \%$ paraformaldehyde followed by sonication ( 1.5 cycles, $70 \%$ amplitude) to shear the gDNA. Sonicated lysates were pulled 


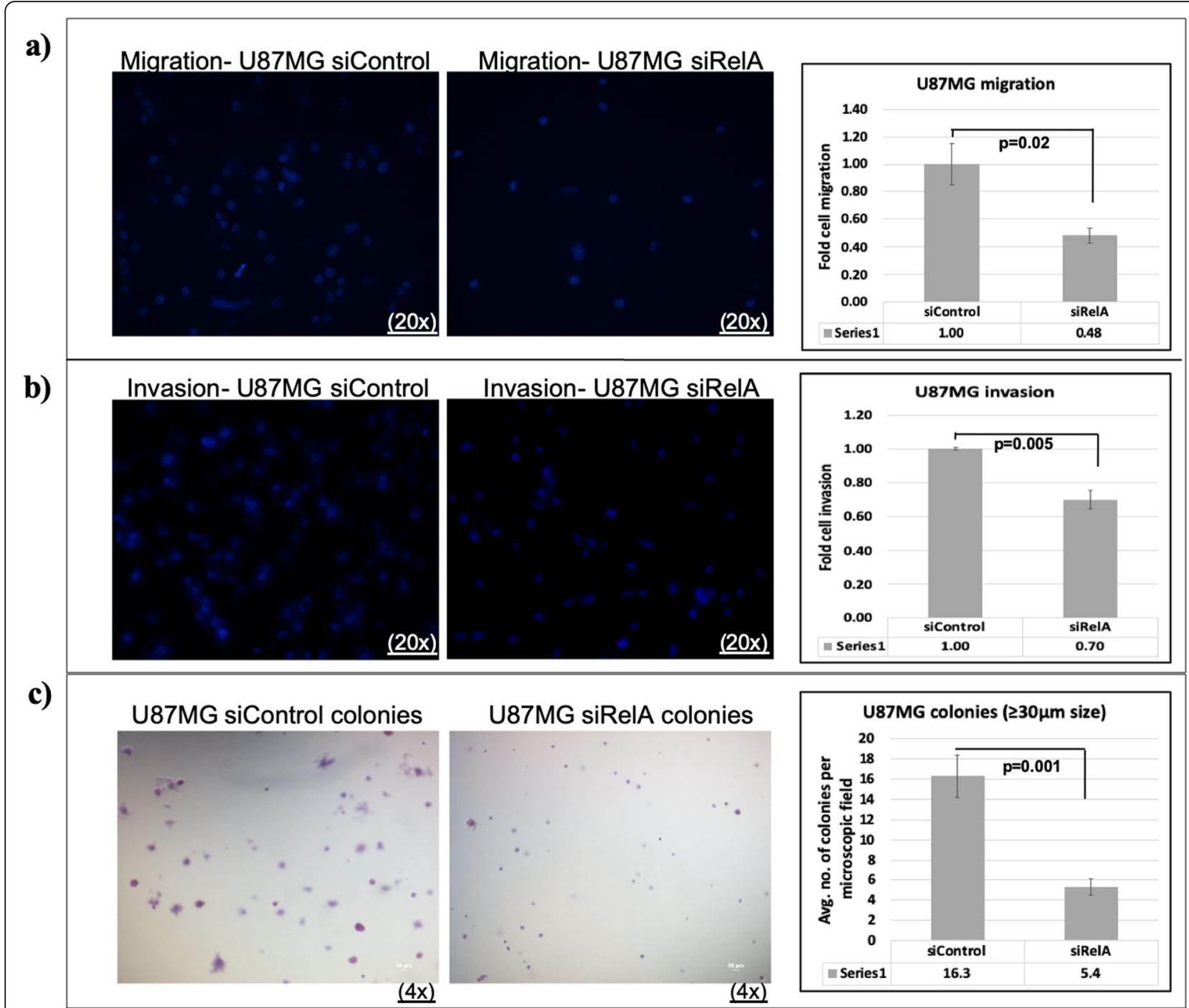

Fig. 8 Effect of NFKB (RelA) knockdown on migration, invasion and clonogenicity of U87MG cells. Representative images (20x magnification) and quantification of (a) cell migration and (b) cell invasion in U87MG after NFKB (RelA) knockdown are shown. Transwell cell migration and invasion (matrigel) assays were performed for 24 and $48 \mathrm{~h}$ respectively, using U87MG cells harvested after $48 \mathrm{~h}$ of siRelA/siControl transfection. Cells that migrated across transwell membrane or invaded through matrigel were fixed, stained and counted. Mean of five non-overlapping fields was calculated in each of the triplicate wells. Number of cells that migrated/invaded in siRelA cells were normalized against the number of cells that migrated/invaded in siControl cells. Values in graphs are expressed as fold change. Significant decrease in migration and invasion was observed in siRelA-treated U87MG cells as compared to the siControl cells as determined by Student's t-test. c Anchorage-independent colony formation assay. Representative micrographs (4x magnification; scale bar: $50 \mu \mathrm{m}$ ) and quantification of colonies in soft agar assay of U87MG after NFkB (RelA) knockdown are shown. U87MG cells were harvested after $48 \mathrm{~h}$ of siRelA/siControl transfection and 15,000 cells/well were seeded in 0.3\% agarose in DMEM supplemented with 20\% FBS in triplicate wells in a six-well plate. After 22 days, colonies were stained with crystal violet. Mean number of colonies ( $\geq 30 \mu \mathrm{m}$ size) of ten non-overlapping fields was calculated in each of the triplicate wells in siRelA/siControl cells. Graph values are expressed as mean \pm s.d. Significant difference was observed in siRelA-treated U87MG cells as compared to the siControl cells as determined by Student's $t$-test

down with specific antibodies against NFkB (RelA), pol-II (positive control) and IgG (negative control). After reverse cross-linking of pulled-down DNA and protein, PCR amplification of pulled-down DNA was done using primers (Additional file 1: Table S3) designed to amplify flanking region of NFkB (RelA) motif (at $-90 \mathrm{bp} /-80 \mathrm{bp}$ ) on FAT1 promoter corresponding to $190 \mathrm{bp}$ product. Gel electrophoresis analysis confirms the presence of specific ChIP product at $190 \mathrm{bp}$ size (Fig. $7 \mathrm{~d}$ ), confirming the presence of NFKB (RelA) motif (at $-90 \mathrm{bp} /-80 \mathrm{bp}$ ) and the cooperative binding of NFKB (RelA) to endogenous FAT1 promoter.

\section{Reduced promoter activity of the mutated FAT1} promoter (pGL3F1mtRelA) as well as positive ChIP assay finding of endogenous $N F K B$ (RelA) binding to the motif $[-90 b p /-80 b p$ wrt TSS(+1)] therefore, confirm 
the significance of transcription factor $N F \kappa B$ (RelA)

binding to the FAT1 promoter and its role in regulating FAT1 transcription.

\section{NFkB (RelA) silencing decreases cell migration, cell invasion and anchorage-independent colony formation capacity in U87MG cells}

In order to confirm that the increase in migration, invasion and colony forming capacity of GBM cells mediated by FAT $1[17,19]$ occurs under the transcriptional control of NFkB (RelA), we transfected U87MG cells with NFkB (RelA) specific siRNA followed by in-vitro transwell migration and invasion assays and soft agar colony formation assay. U87MG cells were harvested $48 \mathrm{~h}$ post-siRNA transfection and subjected to transwell migration for $24 \mathrm{~h}$ as well as transwell matrigel invasion for $48 \mathrm{~h}$. We found $52 \%$ decrease in the migration of siRelA U87MG cells (Fig. 8a) and 30\% decrease in the invasion of siRelA U87MG cells (Fig. 8b) as compared to their respective siControl treated cells.

U87MG cells were cultured and transfected with NFkB (RelA) specific siRNA. After $48 \mathrm{~h}$ of transfection, the cells were transferred in anchorage-independent soft agar medium and cultured for 22 days. A significant decrease in the size of the colonies formed in siRelA treated cells was found as compared to siControl treated cells (Fig. 8c). On counting the colonies of size $\geq 30 \mu \mathrm{m}$ in both siControl and siRelA treated cells, we observed an average $16.3( \pm 2.1)$ colonies per field in the case of siControl cells and only $5.4( \pm 0.8)$ colonies per field in siRelA cells (Fig. 8c). Hence, NFkB (RelA) knockdown, similar to FAT1 knockdown [17, 19], inhibits cell migration, invasion and clonogenic ability in GBM cells, reflecting the importance of NFkB-FAT1 axis and their tumorigenic potential in GBM.

\section{Discussion}

The FAT1 gene located on chromosome 4q35.2 encodes for FAT1, a large $506 \mathrm{kDa}$ transmembrane protein, belonging to cadherin superfamily [21]. Functional evidence suggests that FAT1 plays an important role in various biological processes, including developmental processes [34], cell-cell communication [20, 22], adhesion and migration [35-37]. In cancers, FAT1 is found to have oncogenic $[17-19,23-26,31]$ as well as tumor suppressive role $[27,38]$ in context dependent manner. FAT1 expression is found to be upregulated in different cancers like pancreatic cancer [26], hepatocellular carcinoma [25], B-cell acute lymphoblastic leukemia [23], colon cancer [24] and glioma [17-19, 31] suggesting its oncogenic function in these cancers.

We have observed increased expression and oncogenic effect of FAT1 in GBM by upregulating pro-inflammatory molecules [17] as well as by promoting HIF-1 $\alpha$ expression and signaling under hypoxia [18]. In addition, FAT1 has also been found to be essential for maintaining the clonogenic capacity of GBM cells by substantially upregulating expression of EMT/stemness markers under hypoxia [19]. The clonogenic effect of FAT1 has also been reported in U251 glioma cells [31]. The critical role of FAT1 in maintaining pro-tumorigenic microenvironment responsible for tumor progression and aggressiveness in GBM has been evident from the above reports. Furthermore, studies on FAT1 till date are mainly focused on its overexpression in tumors and its downstream functional outcome [17$19,25,38]$. However, the upstream transcriptional regulators crucial for FAT1 expression have not been reported in literature. This study identifies and addresses the crucial role of NFкB (RelA) in the transcriptional regulation of FAT1 in GBM.

On in-silico promoter analysis, we observed binding motifs for various known transcription factors including NFкB-family on FAT1 promoter ( $-3220 \mathrm{bp}$ to $+848 \mathrm{bp}$, w.r.t. TSS + 1). NFkB (nuclear factor kappa light chain enhancer of activated B cells) is a potent transcription factor that controls transcription of a number of genes involved in inflammation, cell proliferation, invasion/metastasis etc. leading to tumor aggressiveness $[9,10,39]$. NFKB (RelA) is documented to be highly active in GBM $[10,11]$ and known to upregulate inflammatory microenvironment $[15,33]$ and tumor aggressiveness in GBM [10]. NFKBpathway is known to be induced by tumor necrosis factor (TNF $\alpha$ ), hypoxia, bacterial lipopolysaccharides, inflammatory stimuli etc. $[15,16,40]$. Constitutively active $\mathrm{NFK}_{\mathrm{K}}$ is seen in GBM and inhibition of NFкB-activity induces apoptosis [41]. NFкB-activity has also been associated with comparatively short survival in glioma patients [42].

We observed seventeen (17) binding motifs for transcription factor NFкB (RelA) on FAT1 promoter with high probability matrix score, suggesting a possible functional link between NFKB (RelA) and FAT1. Previous reports suggested that GBM is characterized with upregulated expression of FAT1 [17-19] and NFкB (RelA) [11, 14]. Here, observation of significant increased expression and positive correlation between FAT1 and NFкB (RelA) expression in our GBM samples $(r=0.70 ; p=0.002)$ further strengthened this novel link between FAT1 and NFкB (RelA) expression. Tumors expressing high NFkB showed high FAT1 expression and tumors with low NFkb expression showed low FAT1. However, few samples showed high FAT1 with low NFkB expression which could be due to the regulation of FAT1 expression by transcription factors (as identified by in-silico promo software analysis) other than NFкB (RelA). However, we have not studied the clinical features of these tumors. The association of this group with any clinical 
phenotype needs further study in future. In addition, positive correlation between FAT1 and NFкB (RelA) expression was also observed in REMBRANDT-GBMdataset $(r=0.276 ; p=0.00004)$ as well as in TCGA GBM-dataset $(r=0.213, p=0.008)$. The possible reason for the lower $r$ value observed in the TCGA and Rembrandt GBM could be due to the outlier values in the bigger sample size. Similar observation of positive expression correlation between NFKB (RelA) and FAT1 in other non-glioma tumors like pancreatic, hepatocellular, stomach and lung tumors suggests the significance of NFкB (RelA)-FAT1 link in other tumors as well.

In order to confirm the transcriptional role of NFKB (RelA) on the expression of FAT1 in GBM, we have cloned and characterized $4.0 \mathrm{~kb}$ FAT1-promoter $p G L 3 F 4$ $[-3220$ bp to +848 bp, w.r.t. TSS $(+1)$ having $17 \mathrm{NFKB}$ (RelA) sites] and different FAT1 promoter-deletion constructs [ $p G L 3 F 3(3.1 \mathrm{~kb}), p G L 3 F 2(2.1 \mathrm{~kb}), p G L 3 F 1$ (1.0 $\mathrm{kb}), p G L 3 F 1 \delta 1$ (0.86 kb), $p G L 3 F 1 \delta 2(0.25 \mathrm{~kb})]$. Among all promoter constructs generated and analyzed, $p G L 3 F 1$ [1 $\mathrm{kb} ;-200 \mathrm{bp}$ to +848 bp, w.r.t. TSS $(+1)$ with 3 -NFkB (RelA) binding sites (at $-90 \mathrm{bp} /-80 \mathrm{bp},+347 \mathrm{bp} /+356 \mathrm{bp}$ and $+360 \mathrm{bp} /+368 \mathrm{bp}$ ) showed the highest promoter activity in GBM cells (U87MG/A172/U373MG) reflecting the potentially strongest site for $\mathrm{NFKB}$ (RelA) activity. The promoter (pGL3F1) deletion analysis indicated NFKB (RelA) site at $-90 \mathrm{bp} /-80 \mathrm{bp}$ on FAT1 promoter to be critical for NFкB (RelA) activity.

And since hypoxia and TNF $\alpha$ are known activators of NFKB pathways in GBM [30, 33], on exposure of $p G L 3 F 1$ FAT1-promoter transfected GBM cells to severe hypoxia $\left(0.2 \% \mathrm{O}_{2}\right)$, TNF $\alpha$ and NFкB (RelA) (RelA+IKBK overexpressing vectors) increased the $p G L 3 F 1$-promoter activity. Significantly increased levels of endogenous-FAT1 transcripts and FAT1 protein level were also observed. Furthermore, siRNA-knockdown of NFkB (RelA) significantly downregulated the FAT1 mRNA-expression, FAT1 promoter activity as well as reduction in FAT1 protein level. Moreover, 5' deletion of NFKB (RelA) motif at $-90 \mathrm{bp} /-$ $80 \mathrm{bp}$ from pGL3F1 as well as site directed mutagenesis (pGL3F1mtRelA) of NFкB (RelA) motif at $-90 \mathrm{bp} /-80 \mathrm{bp}$ from pGL3F1 resulted in significant reduction in the promoter activity. In addition, the endogenous binding of NFKB (RelA) at $-90 \mathrm{bp} /-80 \mathrm{bp}$ on FAT1 promoter was also confirmed by ChIP assay. These findings validated and confirmed NFKB (RelA) as a potential transcription factor regulating FAT1 expression in GBM.

Our findings signify the regulatory importance of NFKB (RelA) on FAT1 mRNA expression. NFkB is one of the master regulators of pro-inflammatory genes in cancer which upregulates inflammatory-microenvironment in cancer by increasing the expression of cytokines and chemokines $[15,16]$ as well as in regulating cell proliferation, apoptosis, cell-morphogenesis, cell-differentiation etc. in cell/context dependent manner [9-13, 16]. Hypoxia has long been identified to co-exist with chronic inflammation in tumors $[15,16,28,29]$ and HIF- $1 \alpha$, a master regulator of tumor hypoxia, is known to be regulated in NFKB dependent manner and cooperatively maintain malignant phenotypes in cancer $[16,43,44]$. We had initially demonstrated that FAT1 is a major contributor to protumorigenic inflammation in gliomas driving the upregulation of pro-inflammatory cytokines like IL6 and IL1ß and also COX2 via AP1 activation [17]. Subsequently, we have also shown FAT1 to up-regulate HIF1 $\alpha$ at the level of both transcription and translation via the AKT/mTOR pathway [18], and simultaneously increasing cellular invasion. We have also demonstrated FAT1 to affect EMT and stemness, both independently and also via HIF1 $\alpha$ in GBM under hypoxia [19]. Interestingly, activated NFкB also contributes to epithelial-mesenchymal transition and promote invasion/metastasis [9]. Tumor cells with activated NFKB contribute to maintenance of stemness in different tumors [16, 45-47]. On knocking down NFкB (RelA) in GBM cells, a significant decrease in the migration, invasion and colony forming capacity of U87MG cells was observed. Interesting parallels between the activity of NFKB and FAT1 have been demonstrated, at least in glioma. However, as NFKB (RelA) is known to affect several distinct downstream pathways, the exact contribution of the NFкB-FAT1 axis cannot be determined by this study and is beyond the scope of this work. Future studies are needed to validate the extent of this axis. The positive correlation of FAT1 and NFKB (RelA) in other tumors like pancreatic, hepatocellular, lung and stomach cancers suggests that the same mechanism may be operative in these tumors also. However, this is yet to be confirmed experimentally. The role of NFKB regulated pathways in maintaining and promoting hypoxia, inflammation and stemness in cancer cells has been an area of significant scientific interest and many NFKB/HIF- $1 \alpha$ inhibitors are under clinical cancer trials [48-51]. Our results regarding the role of NFKB (RelA) in upregulating FAT1 transcription and in regulating cell migration/invasion and clonogenecity support the possibility that the pro-tumorigenic responses by NFrB (RelA) may be partly mediated through the NFkB-FAT1 axis.

\section{Conclusion}

This study shows a novel link between NFкB (RelA) and FAT1 at the molecular level. Hence, we suggest that the NFкB-FAT1 axis may have clinical relevance as a potential therapeutic target for combating protumorigenic inflammation, hypoxic response, stemness and EMT related phenotypes. While this study has been conducted in GBM, this axis may be relevant in other tumors as well. 


\section{Supplementary information}

Supplementary information accompanies this paper at https://doi.org/10. 1186/s12885-019-6435-1

Additional file 1: Figure S1. BLAST alignment of FAT1 gDNA sequence and FAT1 transcript sequence. Figure S2. In-silico analysis of FAT1 promoter sequence showing binding motifs for potential transcription factors. Figure S3. In-silico analysis of FAT1 promoter sequence showing (a) partial sequence of the FAT1 promoter region $-3220 \mathrm{bp}$ to $+848 \mathrm{bp}$ W.r.t. TSS (+1) with binding motifs for potential transcription factors and (b) NFKB (RelA)/RelA binding sites and dissimilarity rate (matrix score) on FAT1 promoter (4.0kb). Figure S4. Correlation of NFKB (RelA) and FAT1 expression in different tumors from Rembrandt database. Figure S5. Output of nucleotide BLAST search, performed using 4.0kb putative FAT1 upstream region. Figure S6. In-silico analysis of core promoter region of FAT1 gene. Figure S7. Luciferase activity of $5^{\prime}$ deletion constructs of FAT1 promoter constructs (pGL3F2): U87MG cells were transfected with pGL3F2 (2.1 Kb) and its 5'Deletion constructs [pGL3F2 81 (1.3kb) and pGL3F2 82 (1.1 kb)] along with pGL3F1 for 48 hours. Figure S8. a) Gradient PCR (annealing temperature range $-50^{\circ} \mathrm{C}$ to $70.5^{\circ} \mathrm{C}$ ) was performed to confirm the annealing temperature of NFkB (RelA) SDM primers. PCR amplified product of mutated construct (pGL3F1mtRelA) was observed at the annealing temperature at $69.7^{\circ} \mathrm{C}$ (Lane 3$), 68.1^{\circ} \mathrm{C}$ (Lane 4) and $66^{\circ} \mathrm{C}$ (Lane 5) by gel electrophoresis analysis. b) pGL3F1 [wild type NFkB (RelA) site] was taken as parental plasmid to amplify muatated consruct (pGL3F1mtRelA), further parental plasmid was digested with Dpnl restriction enzyme followed by gel electrophoresis analysis. Figure S9. Confirmation of NFkB (RelA) mutation in pGL3F1mtRelA construct by EcoRI digestion and sequencing analysis. Table S1. List of PCR Primers. Table S2. List of various FAT1 promoter constructs. Table S3. Nucleotide sequence of PCR primers used for $\mathrm{QPCR}$ ChIP assay and site directed mutagenesis.

\section{Abbreviations}

ChIP: Chromatin-immunoprecipitation; EMT: Epithelial-Mesenchyma Transition; GBM: Glioblastoma; HIF-1a: Hypoxia Inducing Factor- 1a; NFkB: Nuclear Factor k B; TNFa: Tumor Necrosis factor a; TSS: Transcription Start Site

\section{Acknowledgements}

We would like to acknowledge Prof. Soumen Basak, NII, New Delhi, India for kindly providing ReIA and IKBK expression vectors. We thank Ms. Jyoti, Mr. Manoj and Mr. Pappu for technical and administrative help. We thank Mr. Jitender Bihari Lal and Mr. Mukesh for their assistance in routine lab work and cell culture work.

\section{Authors' contributions}

CS, KI, YG performed the experiments, result analysis and data acquisition. C Sarkar performed the histological diagnosis and classification of patient samples. AS performed surgery and provided clinical samples. PC, SS, KC contributed to study design, manuscript preparation and editing, data evaluation, funding \& resources. All the authors have read and approved the final manuscript.

\section{Funding}

The work has been financially supported by grants from Indian Council of Medical Research (ICMR), India [No.5/13/58/2014/NCD-III] and Department of biotechnology (DBT), India [No.BT/PR13357/MED/30/1532/2015] to KC; JC Bose fellowship of DST (Department of Science and technology) India to SS; AllMS institute fellowship to CS; DST-SERB Start-Up fellowship to Kl; AlIMS institute fellowship and ICMR fellowship to YG. The funding body played no role in the design of the study and collection, analysis, interpretation of data and in writing the manuscript.

\section{Availability of data and materials}

All data generated or analysed during this study and materials used are available in the manuscript and supplementary information file.

\section{Ethics approval and consent to participate}

-Surgically resected human GBM samples were collected with due patient written consent after approval by Institute Ethics-Committee, AlIMS, New Delhi (Ref. No IESC/T-416/01.11.2013).

-ATCC cell lines used in this study have been authenticated by STR cell identification (Project ID: M-1070, Lifecode, New Delhi) on 12th, July, 2016. Cell lines were not infected by mycoplasma.

-Approval for use of the cell lines for in-vitro study was received from Institutional Committee on Bio-safety for Recombinant DNA Research, AlIMS, New Delhi, dated 6th April 2015.

\section{Consent for publication}

Not Applicable.

\section{Competing interests}

The authors declare that they have no conflict of interest.

\section{Author details}

${ }^{1}$ Department of Biochemistry, All India Institute of Medical Sciences, -110029, New Delhi, India. ${ }^{2}$ Present address: Cell Biology, National Institute of Environmental Health Sciences, National Institutes of Health, Durham, NC 27709, USA. ${ }^{3}$ Department of Pathology, All India Institute of Medical Sciences, New Delhi, India. ${ }^{4}$ Department of Neurosurgery, All India Institute of Medical Sciences, New Delhi, India.

Received: 4 April 2019 Accepted: 5 December 2019

Published online: 28 January 2020

\section{References}

1. Gilbertson RJ, Rich JN. Making a tumour's bed: glioblastoma stem cells and the vascular niche. Nat Rev Cancer. 2007;7:733-6.

2. Louis DN, Perry A, Reifenberger G, von Deimling A, Figarella-Branger D, Cavenee WK, et al. The 2016 World Health Organization classification of tumors of the central nervous system: a summary. Acta Neuropathol (Berl). 2016;131:803-20.

3. Martínez-González A, Calvo GF, Pérez Romasanta LA, Pérez-García VM. Hypoxic cell waves around necrotic cores in Glioblastoma: a biomathematical model and its therapeutic implications. Bull Math Biol. 2012;74:2875-96.

4. Stupp R, Mason WP, van den Bent MJ, Weller M, Fisher B, Taphoorn MJB, et al. Radiotherapy plus concomitant and adjuvant temozolomide for glioblastoma. N Engl J Med. 2005;352:987-96.

5. Irshad K, Mohapatra SK, Srivastava C, Garg H, Mishra S, Dikshit B, et al. A combined gene signature of hypoxia and notch pathway in human glioblastoma and its prognostic relevance. PLoS One. 2015;10:e0118201.

6. Kim Y-W, Koul D, Kim SH, Lucio-Eterovic AK, Freire PR, Yao J, et al. Identification of prognostic gene signatures of glioblastoma: a study based on TCGA data analysis. Neuro-Oncol. 2013;15:829-39.

7. Kitange GJ, Templeton KL, Jenkins RB. Recent advances in the molecular genetics of primary gliomas. Curr Opin Oncol. 2003;15:197-203.

8. Marampon F, Gravina GL, Zani BM, Popov VM, Fratticci A, Cerasani M, et al. Hypoxia sustains glioblastoma radioresistance through ERKs/DNA-PKcs/HIFla functional interplay. Int J Oncol. 2014;44:2121-31.

9. Rinkenbaugh AL, Cogswell PC, Calamini B, Dunn DE, Persson Al, Weiss WA, et al. IKK/NF-KB signaling contributes to glioblastoma stem cell maintenance. Oncotarget. 2016;7:69173-87.

10. Raychaudhuri B, Han Y, Lu T, Vogelbaum MA. Aberrant constitutive activation of nuclear factor kappaB in glioblastoma multiforme drives invasive phenotype. J Neuro-Oncol. 2007;85:39-47.

11. Friedmann-Morvinski D, Narasimamurthy R, Xia Y, Myskiw C, Soda Y, Verma IM. Targeting NF-kB in glioblastoma: a therapeutic approach. Sci Adv. 2016; 2:e1501292.

12. Cahill KE, Morshed RA, Yamini B. Nuclear factor-KB in glioblastoma: insights into regulators and targeted therapy. Neuro-Oncol. 2016;18:329-39.

13. Nagai S, Washiyama K, Kurimoto M, Takaku A, Endo S, Kumanishi T. Aberrant nuclear factor-kappaB activity and its participation in the growth of human malignant astrocytoma. J Neurosurg. 2002;96:909-17.

14. Nogueira L, Ruiz-Ontañon P, Vazquez-Barquero A, Moris F, Fernandez-Luna $J$. The NFKB pathway: a therapeutic target in glioblastoma. Oncotarget. 2011;2:646-53. 
15. Oliver KM, Taylor CT, Cummins EP. Hypoxia. Regulation of NFkB signalling during inflammation: the role of hydroxylases. Arthritis Res Ther. 2009;11:215.

16. Tafani M, Di Vito M, Frati A, Pellegrini L, De Santis E, Sette G, et al. Proinflammatory gene expression in solid glioblastoma microenvironment and in hypoxic stem cells from human glioblastoma. J Neuroinflammation. 2011;8:32.

17. Dikshit B, Irshad K, Madan E, Aggarwal N, Sarkar C, Chandra PS, et al. FAT1 acts as an upstream regulator of oncogenic and inflammatory pathways, via PDCD4, in glioma cells. Oncogene. 2013;32:3798-808.

18. Madan E, Dikshit B, Gowda SH, Srivastava C, Sarkar C, Chattopadhyay P, et al. FAT1 is a novel upstream regulator of HIF1a and invasion of high grade glioma. Int J Cancer. 2016;139:2570-82.

19. Srivastava C, Irshad K, Dikshit B, Chattopadhyay P, Sarkar C, Gupta DK, et al. FAT1 modulates EMT and stemness genes expression in hypoxic glioblastoma. Int J Cancer. 2017;142(4):805-12.

20. Badouel C, Zander MA, Liscio N, Bagherie-Lachidan M, Sopko R, Coyaud E, et al. Fat1 interacts with Fat4 to regulate neural tube closure, neural progenitor proliferation and apical constriction during mouse brain development. Dev Camb Engl. 2015;142:2781-91.

21. Dunne J, Hanby AM, Poulsom R, Jones TA, Sheer D, Chin WG, et al. Molecular cloning and tissue expression of FAT, the human homologue of the Drosophila fat gene that is located on chromosome 4q34-q35 and encodes a putative adhesion molecule. Genomics. 1995;30:207-23.

22. Tanoue T, Takeichi M. Mammalian Fat1 cadherin regulates actin dynamics and cell-cell contact. J Cell Biol. 2004;165:517-28.

23. de Bock CE, Ardjmand A, Molloy TJ, Bone SM, Johnstone D, Campbell DM, et al. The Fat1 cadherin is overexpressed and an independent prognostic factor for survival in paired diagnosis-relapse samples of precursor B-cell acute lymphoblastic leukemia. Leukemia. 2012;26:918-26.

24. Pileri P, Campagnoli S, Grandi A, Parri M, De Camilli E, Song C, et al. FAT1: a potential target for monoclonal antibody therapy in colon cancer. $\mathrm{Br} J$ Cancer. 2016;115:40-51.

25. Valletta D, Czech B, Spruss T, Ikenberg K, Wild P, Hartmann A, et al. Regulation and function of the atypical cadherin FAT1 in hepatocellular carcinoma. Carcinogenesis. 2014;35:1407-15.

26. Wojtalewicz N, Sadeqzadeh E, Weiß JV, Tehrani MM, Klein-Scory S, Hahn S, et al. A soluble form of the giant cadherin Fat1 is released from pancreatic cancer cells by ADAM10 mediated ectodomain shedding. PLoS One. 2014;9:e90461.

27. Nishikawa Y, Miyazaki T, Nakashiro K-I, Yamagata H, Isokane M, Goda H, et al. Human FAT1 cadherin controls cell migration and invasion of oral squamous cell carcinoma through the localization of $\beta$-catenin. Oncol Rep. 2011;26:587-92.

28. Chandel NS, Trzyna WC, McClintock DS, Schumacker PT. Role of oxidants in NF-kappa B activation and TNF-alpha gene transcription induced by hypoxia and endotoxin. J Immunol Baltim Md 1950. 2000;165:1013-21

29. Leeper-Woodford SK, Detmer K. Acute hypoxia increases alveolar macrophage tumor necrosis factor activity and alters NF-kappaB expression. Am J Phys. 1999:276(6 Pt 1):L909-16.

30. Xia Y, Shen S, Verma IM. NF-KB, an active player in human cancers. Cancer Immunol Res. 2014:2:823-30.

31. Kranz D, Boutros M. A synthetic lethal screen identifies FAT1 as an antagonist of caspase-8 in extrinsic apoptosis. EMBO J. 2014;33:181-97.

32. Kang M-H, Jeong GS, Smoot DT, Ashktorab H, Hwang CM, Kim BS, et al. Verteporfin inhibits gastric cancer cell growth by suppressing adhesion molecule FAT1. Oncotarget. 2017;8:98887-97.

33. Culver C, Sundqvist A, Mudie S, Melvin A, Xirodimas D, Rocha S. Mechanism of hypoxia-induced NF-KB. Mol Cell Biol. 2010;30:4901-21.

34. Bryant PJ, Huettner B, Held LI, Ryerse J, Szidonya J. Mutations at the fat locus interfere with cell proliferation control and epithelial morphogenesis in Drosophila. Dev Biol. 1988;129:541-54.

35. Hou R, Liu L, Anees S, Hiroyasu S, Sibinga NES. The Fat1 cadherin integrates vascular smooth muscle cell growth and migration signals. J Cell Biol. 2006; 173:417-29.

36. Moeller MJ, Soofi A, Braun GS, Li X, Watzl C, Kriz W, et al. Protocadherin FAT1 binds EnaNASP proteins and is necessary for actin dynamics and cell polarization. EMBO J. 2004;23:3769-79.

37. Schreiner D, Müller K, Hofer HW. The intracellular domain of the human protocadherin hFat1 interacts with Homer signalling scaffolding proteins. FEBS Lett. 2006:580:5295-300.

38. Morris LGT, Kaufman AM, Gong Y, Ramaswami D, Walsh LA, Turcan \$̧, et al. Recurrent somatic mutation of FAT1 in multiple human cancers leads to aberrant Wnt activation. Nat Genet. 2013;45:253-61.
39. Karin M, Greten FR. NF-kappaB: linking inflammation and immunity to cancer development and progression. Nat Rev Immunol. 2005;5:749-59.

40. Qin H, Wilson CA, Lee SJ, Zhao X, Benveniste EN. LPS induces CD40 gene expression through the activation of NF-kappaB and STAT-1alpha in macrophages and microglia. Blood. 2005;106:3114-22.

41. Sen E. Targeting inflammation-induced transcription factor activation: an open frontier for glioma therapy. Drug Discov Today. 2011;16:1044-51.

42. Bredel M, Scholtens DM, Yadav AK, Alvarez AA, Renfrow JJ, Chandler JP, et al. NFKBIA deletion in glioblastomas. N Engl J Med. 2011;364:627-37.

43. Rius J, Guma M, Schachtrup C, Akassoglou K, Zinkernagel AS, Nizet V, et al. NF-kappaB links innate immunity to the hypoxic response through transcriptional regulation of HIF-1alpha. Nature. 2008;453:807-11.

44. van Uden P, Kenneth NS, Rocha S. Regulation of hypoxia-inducible factor1alpha by NF-kappaB. Biochem J. 2008;412:477-84.

45. Garner JM, Fan M, Yang CH, Du Z, Sims M, Davidoff AM, et al. Constitutive activation of signal transducer and activator of transcription 3 (STAT3) and nuclear factor $\mathrm{KB}$ signaling in glioblastoma cancer stem cells regulates the notch pathway. J Biol Chem. 2013;288:26167-76.

46. Rajasekhar VK, Studer L, Gerald W, Socci ND, Scher HI. Tumour-initiating stem-like cells in human prostate cancer exhibit increased NF-KB signalling. Nat Commun. 2011;2:162.

47. Wang H, Lathia JD, Wu Q, Wang J, Li Z, Heddleston JM, et al. Targeting interleukin 6 signaling suppresses glioma stem cell survival and tumor growth. Stem Cells Dayt Ohio. 2009:27:2393-404.

48. Semenza GL. Hypoxia-inducible factors: mediators of cancer progression and targets for cancer therapy. Trends Pharmacol Sci. 2012;33:207-14.

49. Xia Y, Choi H-K, Lee K. Recent advances in hypoxia-inducible factor (HIF)-1 inhibitors. Eur J Med Chem. 2012:49:24-40.

50. Lin Y, Bai L, Chen W, Xu S. The NF-kB activation pathways, emerging molecular targets for cancer prevention and therapy. Expert Opin Ther Targets. 2010;14:45-55.

51. Shen H-M, Tergaonkar V. NFkappaB signaling in carcinogenesis and as a potential molecular target for cancer therapy. Apoptosis Int J Program Cell Death. 2009;14:348-63.

\section{Publisher's Note}

Springer Nature remains neutral with regard to jurisdictional claims in published maps and institutional affiliations.
Ready to submit your research? Choose BMC and benefit from:

- fast, convenient online submission

- thorough peer review by experienced researchers in your field

- rapid publication on acceptance

- support for research data, including large and complex data types

- gold Open Access which fosters wider collaboration and increased citations

- maximum visibility for your research: over $100 \mathrm{M}$ website views per year

At $\mathrm{BMC}$, research is always in progress.

Learn more biomedcentral.com/submissions 